Article

\title{
Total and Free Sugar Content of Canadian Prepackaged Foods and Beverages
}

\author{
Jodi T. Bernstein ${ }^{1}$, Alyssa Schermel ${ }^{1}$, Christine M. Mills ${ }^{2}$ and Mary R. L'Abbé ${ }^{1, *}$ \\ 1 Department of Nutritional Sciences, Faculty of Medicine, University of Toronto, Toronto, ON M5S 3E2, \\ Canada; jodi.bernstein@mail.utoronto.ca (J.T.B.); a.schermel@gmail.ca (A.S.) \\ 2 Dalla Lana School of Public Health, University of Toronto, Toronto, ON M5T 3M7, Canada; \\ chris.mills@mail.utoronto.ca \\ * Correspondence: mary.labbe@utoronto.ca; Tel.: +1-416-978-7235
}

Received: 25 July 2016; Accepted: 12 September 2016; Published: 21 September 2016

\begin{abstract}
A number of recommendations for policy and program interventions to limit excess free sugar consumption have emerged, however there are a lack of data describing the amounts and types of sugar in foods. This study presents an assessment of sugar in Canadian prepackaged foods including: (a) the first systematic calculation of free sugar contents; (b) a comprehensive assessment of total sugar and free sugar levels; and (c) sweetener and free sugar ingredient use, using the University of Toronto's Food Label Information Program (FLIP) database $2013(n=15,342)$. Food groups with the highest proportion of foods containing free sugar ingredients also had the highest median total sugar and free sugar contents (per $100 \mathrm{~g} / \mathrm{mL}$ ): desserts $(94 \%, 15 \mathrm{~g}$, and $12 \mathrm{~g}$ ), sugars and sweets $(91 \%$, $50 \mathrm{~g}$, and $50 \mathrm{~g})$, and bakery products $(83 \%, 16 \mathrm{~g}$, and $14 \mathrm{~g}$, proportion with free sugar ingredients, median total sugar and free sugar content in Canadian foods, respectively). Free sugar accounted for $64 \%$ of total sugar content. Eight of 17 food groups had $\geq 75 \%$ of the total sugar derived from free sugar. Free sugar contributed $20 \%$ of calories overall in prepackaged foods and beverages, with the highest at $70 \%$ in beverages. These data can be used to inform interventions aimed at limiting free sugar consumption.
\end{abstract}

Keywords: sugars; free sugar; nutrition labelling; food composition; food supply; Canada; public health; policy

\section{Introduction}

Excess consumption of free sugar (see Box 1 for definitions) has been associated with increased risk of obesity, cardiovascular disease, diabetes, and dental caries [1-5]. In fact, one study found that increased consumption of refined starches, like free sugar, are second only to trans fats in increasing risk of cardiovascular disease [6]. Thus, guidelines to limit intakes to a maximum of $5 \%-10 \%$ of calories/day [7-11] have emerged in many regions. Recommendations have also been made in Canada and other countries to decrease the affordability, availability, accessibility and exposure to products with excess free sugar $[8,12,13]$. Despite these calls to action, the lack of detailed data on the pervasiveness of sugar in the food environment [14] hinders the development of policies and programs to reduce free sugar consumption and associated health benefits with targeted interventions [15]. 
Box 1. Definitions.

- "Free sugar" is the sugar no longer in its naturally-occurring state (i.e., no longer in whole fruits, vegetables, unsweetened dairy, and grains) and can be consumed as is or incorporated into other foods [9]. Examples include table sugar, syrup, honey, fruit juice and nectars.

- " "Added sugar" is the free sugar that has been added to foods [13], however regulatory definitions vary widely under different jurisdictions, some of which are currently under review [16].

- "Naturally-occurring sugar" is the sugar found naturally within whole foods (i.e., within whole fruits, vegetables, dairy, and some grains) [14].

- "Total sugar" is a combination of free sugar and naturally-occurring sugar and is currently the only type of sugar declared on the Nutrition Facts table (NFt) in Canada [17] and in many jurisdictions [16,18-20].

- "Free Sugar Ingredients" (FSI) are all mono- and disaccharides added to foods as well as those naturally-occurring in honey, fruit juices, and syrups (e.g., sugar, honey, maple syrup, molasses, fruit juice, glucose, fructose, agave, and corn syrup) [9].

- "Sweeteners" are food additives that are used to give products a sweet taste and can include sugar alcohols (e.g., malitol, xylitol, and sorbitol), non-nutritive sweeteners (e.g., aspartame, sucralose, and acesulfame-potassium), cyclamate sweeteners, or saccharin sweeteners [21] and are not considered FSI.

There are very limited data available on the free sugar contents of prepackaged foods and on consumption rates in Canada and globally [14]. This may be in part because free sugar is chemically indistinguishable from naturally-occurring sugar and as a result, contents must be calculated or supplied by food manufacturers. This has contributed to free sugar ingredients (FSI) being considered a "hidden" source of calories as it is not always obvious to consumers that they are present in food [22]. This phenomenon has been noted as a worry of Canadian parents [23]. Additionally, the various definitions used to describe sugar and inconsistencies in their components, make comparisons of food composition and sugar intakes problematic, increases the potential for confusion and misinterpretation and points to the need for uniform terminology [14]. Understanding the main sources and amounts of free sugar in Canadian foods will allow for monitoring trends in product formulations, reformulation efforts by the food industry, and Canadian intakes of free sugar overtime, that would otherwise be virtually impossible to measure.

Canadians consumed an average of $110 \mathrm{~g}$ (21.4\% of calories) of total sugar per day in 2004 [24]. Although that report did not differentiate between total sugar and free sugar, another study used these total sugar intakes to estimate the average added sugar consumption of Canadians at $11 \%-13 \%$ of calories [24,25]. These authors estimated the proportion of total sugar coming from added sugar by assuming each of the top food categories contributed either naturally-occurring sugar or added sugar [25]. These total and added sugar consumption rates were based on food composition information obtained from the Canadian Nutrient File (CNF) database, the national nutrition database maintained by Health Canada [26,27]. However, using the CNF to assess sugar in the food supply poses several challenges, including its lack of scheduled, systematic and comprehensive updating, and its lack of brand-specific data [27]. Such data are required for analyzing a rapidly changing food supply, which can vary widely in free sugar content and the use of sweeteners. In contrast with these earlier assessments, more precise estimates of total, added, and free sugar intakes are needed to inform and evaluate relevant public health initiatives.

To obtain a more accurate assessment of the types and amounts of sugar in the Canadian food supply, data need to be reconciled using comprehensive, current, and accurate food composition data [14] along with systematic calculations of free sugar content. Acknowledging this need, in 2014 the Heart and Stroke Foundation of Canada (HSFC) called upon researchers to quantify the amount of free sugar in the Canadian food supply [8]. The overall purpose of this study is to provide a detailed and systematic evaluation of free sugar contents in a large representative sample of Canadian prepackaged foods that can serve as a benchmark to support and measure public health interventions and monitor free sugar consumption. Specific objectives include: (1) determining the amount of free sugar in Canadian prepackaged foods using a step-by-step decision algorithm tailored for use 
on a large, systematically collected, branded food composition database; (2) assessing total sugar and free sugar contents by food group and by detailed subcategory; and (3) conducting the first comprehensive assessment of the use of free sugar ingredients (FSI) and sweeteners in prepackaged foods and beverages.

\section{Materials and Methods}

\subsection{Food Label Information Program (FLIP) Database}

The Food Label Information Program (FLIP) is a database of Canadian food and beverage package labels by brand name that is updated every three years at the University of Toronto (U of T). The purpose of the FLIP is to provide detailed assessments of the nutrition information found on the labels of food products in the Canadian marketplace, and to monitor changes over time. To date, two phases of the FLIP have been completed. The first phase, with data acquired in 2010/2011 (FLIP 2010), is described elsewhere [28]. The second phase, FLIP 2013, is described in this paper. The FLIP 2013 contains nutrition information for 15,342 unique products. Data collection took a similar approach as the FLIP 2010 with regards to acquiring food information from the top selling grocery retailers, although it was fully digitalized to enhance the ease and efficiency of collection and analysis. Food composition database software (University of Toronto and Dietitians of Canada, Toronto, ON, Canada) (web and mobile) was developed for FLIP 2013 in collaboration with the Dietitians of Canada, resulting in a shorter and more efficient food collection and data processing approach.

\subsubsection{FLIP 2013 Data Collection}

Data acquisition occurred between May and September 2013, and was carried out in the Greater Toronto Area and Ottawa, Ontario, and Calgary, Alberta. Data were collected from major outlets of the four largest grocery chains in Canada (Loblaws, Metro, Sobeys, and Safeway), representing 75.4\% of the grocery retail market share [29]. A Smartphone application was developed and used to scan and store the Universal Product Code (UPC), and to photograph all sides of food and beverage packages, and capture price. By systematically scanning the grocery store shelves, every food product with a Nutrition Facts table (NFt), including all available national and private label brands were collected. Seasonal products (e.g., eggnog, Easter chocolates), Natural Health Products (e.g., supplements), baby/toddler foods, and products that did not have a Canadian NFt (e.g., unpackaged fruits, vegetables) were excluded from the data collection. Food products sold at multiple retailers (such as national brand products) were captured only once. When multiple sizes of a product were available, only one size was sampled, but all flavours and varieties of a product were collected. Information collected for each product included the UPC, company, brand, price, NFt information, ingredients, container size, nutrient content claims, disease risk reduction claims, function claims, front of pack symbols, children's marketing, other claims (e.g., organic, natural, and gluten-free), and date and location of sampling.

\subsubsection{FLIP 2013 Data Processing}

Upon scanning the UPC code, foods that had not already been collected in this phase were automatically assigned a product ID and photos uploaded onto the FLIP website for data processing. The FLIP website allowed for efficient data entry using dropdown menus (e.g., to assign foods to specific categories or to indicate the presence of different nutrition claims), and used Optical Character Recognition technology to automatically extract data from the NFt and ingredients list. The FLIP database, run on a Microsoft SQL server, also enabled users to generate data outputs and reports in Microsoft Excel for further statistical analyses.

Food products were classified under multiple categorization systems. Categorization systems used included Schedule M of the Food and Drug Regulations (B.01.001) [30], as well as Health Canada's sodium categories for guiding benchmark sodium levels [31]. These classification systems were also 
used to create similar systems specific to other nutrients, e.g., trans-fat and the sugar focused food categories used for the present study.

When required, data for some food products were also calculated for the "as consumed" form (e.g., cake mixes, drink powders, and condensed soups) using ESHA Food Processor software and food composition data from the Canadian Nutrient File [32] in order to be comparable to the prepared versions within that particular food category. In addition, for some products, serving grams were converted to millilitres and vice versa for consistency across all products within a food category. The database underwent extensive quality control checks including verification of inputted nutrient contents using Atwater factors and outliers to check for erroneous values, and multiple reviews for $\mathrm{NFt}$, Ingredient Lists, gram to millilitre conversions, and food group categorizations. Excluded from this analysis were meal replacement beverages, which are indicated for special dietary use $(n=55)$, and products with missing total sugar declarations $(n=28)$ for a total of 15,259 products in the present study.

\subsubsection{FLIP 2013 Sugar-Focused Food Categories}

Products were classified into 17 sugar-focused major food groups, including 77 major subcategories, and 207 minor categories. Sugar-focused categories were created based on Schedule M food categories as outlined in the Canadian Food and Drug Regulations [30], as well as Health Canada's sodium-focused categories [33]. These categories were further divided or combined on the basis of sugar and sweetener ingredients, intended use, and food type to ensure categories containing like products.

\subsection{Assessment of Free and Total Sugar Content and Use of Free Sugar Ingredients and Sweeteners}

Free sugar is chemically indistinguishable from naturally-occurring sugar [34]. As there is no declaration of free sugar content on the $\mathrm{NFt}$, an algorithm was developed to derive free sugar contents which was guided by a published, systematic methodology for estimating added sugars $[35,36]$. The $U$ of $\mathrm{T}$ free sugar algorithm steps, to be conducted in sequential order, as well as the proportion of free sugar contents calculated at each step, are outlined in Table 1. For the purpose of this analysis, free sugar ingredients (FSI) refers to any free sugar ingredient that meets the WHO definition for free sugar including sugar, syrup, honey, fruit juices, and other sweetening agents [9]. "Sweeteners", as defined by the Canadian Food Inspection Agency as a food additive that is used to give products a sweet taste and can include sugar alcohols (e.g., malitol, xylitol, and sorbitol), non-nutritive sweeteners (e.g., aspartame, sucralose, and acesulfame-potassium), cyclamate sweeteners, or saccharin sweeteners [21] were not considered FSI. Presence of FSI and sweeteners were identified by searching the Ingredient List of each product and the ingredients required in product preparation as stated on the package. The means and distributions of total sugar content, obtained from the NFt, and of the calculated free sugar content were reported as g per $100 \mathrm{~g}$ or g per $100 \mathrm{~mL}$ (the latter for beverages and desserts), by food group, subcategory, and minor category. Free sugar content was calculated as a percent of total sugar and as a percent of energy, the latter to allow for comparisons with maximum intake guidelines, which are usually presented as a percent of calories. All calculations were conducted on the sugar content of the "as consumed" version of the product.

\subsection{Statistical Analysis}

Mean, SD, and quartiles (min, 25th, 50th, 75th, max) were determined for total sugar and free sugar content. The percent of total sugar and of calories derived from free sugar were presented as proportions. Categorical variables (e.g., presence of FSI and sweetener ingredients) were presented as frequencies (percentages). All statistical analyses were conducted using SAS version 9.4 (SAS Institute Inc., Cary, NC, USA). 
Table 1. Step-by-step method for calculating free sugar content of foods and beverages in the University of Toronto's Food Label Information Program (FLIP) database 2013 and number of foods at each step (total $n=15,259)$.

\begin{tabular}{|c|c|}
\hline Description & $n(\%)^{1}$ \\
\hline $\begin{array}{l}\text { Step 1: Products that contain } 0 \mathrm{~g} \text { total sugar as declared on the NFt. Free sugar } \\
\text { value }=0 \mathrm{~g} / 100 \mathrm{~g} \text {. }\end{array}$ & $3586(23.5 \%)$ \\
\hline $\begin{array}{l}\text { Step 2: Products that contain no FSI }{ }^{2} \text { listed in the Ingredient List. Free sugar } \\
\text { value }=0 \mathrm{~g} / 100 \mathrm{~g} .\end{array}$ & $2620(17.2 \%)$ \\
\hline $\begin{array}{l}\text { Step 3: Products that contain ingredients which contribute no, or a minimal amount of } \\
\text { naturally-occurring sugars (i.e., fruits, vegetables, dairy, grains). Free sugar value }=100 \% \text { of } \\
\text { the declared total sugar content (e.g., soft drinks, fruit drinks) }{ }^{3} \text {. }\end{array}$ & $1642(10.8 \%)$ \\
\hline $\begin{array}{l}\text { Step 4: Products that contain both naturally-occurring sugars and FSI, were compared to } \\
\text { similar products without FSI (from steps } 1 \text { and 2) from the same subcategory (i.e., RTE } \\
\text { breakfast cereals with FSI vs. RTE breakfast cereals without FSI) or minor category (i.e., milk, } \\
\text { flavoured vs. milk, plain). The following equation [36] was used to calculate free } \\
\text { sugar contents: }\end{array}$ & \multirow{3}{*}{$6876(45.1 \%)$} \\
\hline$\frac{100 \times(\text { Sugar per } 100 \mathrm{~g} \text { unsweetened }- \text { Sugar per } 100 \mathrm{~g} \text { sweetened })}{(\text { Sugar per } 100 \mathrm{~g} \text { unsweetened }-100)}$ & \\
\hline $\begin{array}{l}\text { When possible, specific comparisons were made based on main ingredients, flavours, specific } \\
\text { nutrient contents, or product formats (i.e., fat-free sweetened yogurt vs. fat-free plain yogurt). } \\
\text { Calculations resulting in negative free sugar contents }(<0 \mathrm{~g}) \text { were rounded up to } 0 \mathrm{~g} \text {. }\end{array}$ & \\
\hline
\end{tabular}

Step 5: Products that do not have unsweetened comparators in the same subcategory in FLIP, were assigned a free sugar value based on a substitute value from the USDA Database for the Added Sugars Content of Selected Foods [37]. A free sugar value that matches the proportion of total sugar from added sugar in a product from the USDA database was assigned. For example, a product was assigned a free sugar value at $80 \%$ of total sugar if the comparable USDA database product had $80 \%$ of the total sugar coming from added sugars.

Step 6: Products with no comparator in Step 5, were assigned a value reflective of the proportion of total sugar coming from free sugar in products within the same food group (e.g., a chocolate cake is assigned a free sugar value based on the percent of total sugar derived from free sugar content of other products in bakery products). Only products that contained free sugar (steps 3-5) were included in the calculation.

${ }^{1}$ Numbers presented represent the number and proportion (\%) of products calculated at each step. All calculations were done for products in the "as consumed" form. For determination of free sugar contents in the "as consumed" form: total sugar content of the "as consumed" form was used in place of the total sugar content in the "as purchased" form as declared on the NFt; FSI added according to the package directions were treated the same as a FSI in the Ingredient List (Step \#2-6); "as consumed" versions of substitute products were used when available (i.e., brownies instead of brownie mix) (Step \#5); ${ }^{2}$ FSI (free sugar ingredients) for this study refers to all mono- and disaccharides added to foods as well as those naturally-occurring in honey, fruit juices, and syrups (e.g., sugar, honey, maple syrup, molasses, fruit juice, glucose, fructose, agave, and corn syrup) $[9]{ }^{3}$ All products in the energy drinks, fruit drinks, soft drinks, and sports drinks categories that reached Step 3 were considered to contain a minimal amount of naturally-occurring sugars. Abbreviations: FLIP = Food Label Information Program; NFt = Nutrition Facts table; FSI = free sugar ingredients; RTE = Ready-to-eat; USDA = United States Department of Agriculture.

\section{Results}

\subsection{Use of Free Sugar Ingredients and Sweeteners}

Overall, $63.5 \%$ of prepackaged foods contained a FSI, 1.9\% contained a sweetener, another $1.8 \%$ contained both a FSI and a sweetener, and the remaining 32.9\% contained neither (Figure 1). There were 152 unique FSI found in this representative sample of Canadian prepackaged foods, not including variations in spelling, indicators of quality, purity, or origin (e.g., organic maple syrup, 100\% pure agave, and Canadian honey) or specific flavours of fruit juice (e.g., apple juice, and grape juice concentrate) (Table 2). The most common types of FSI found in Canadian food and beverage products were sugar (dried or granulated) identified in $49.3 \%$ of products, glucose in $19.3 \%$, and corn syrup in $10.7 \%$ (Table 2). Major food categories with the highest proportion of products containing FSI were desserts $(93.6 \%)$, sugars and sweets $(91.4 \%)$, and bakery products (83.1\%) (Figure 1). These were also the most total sugar and free sugar dense food groups (Figure 2). 


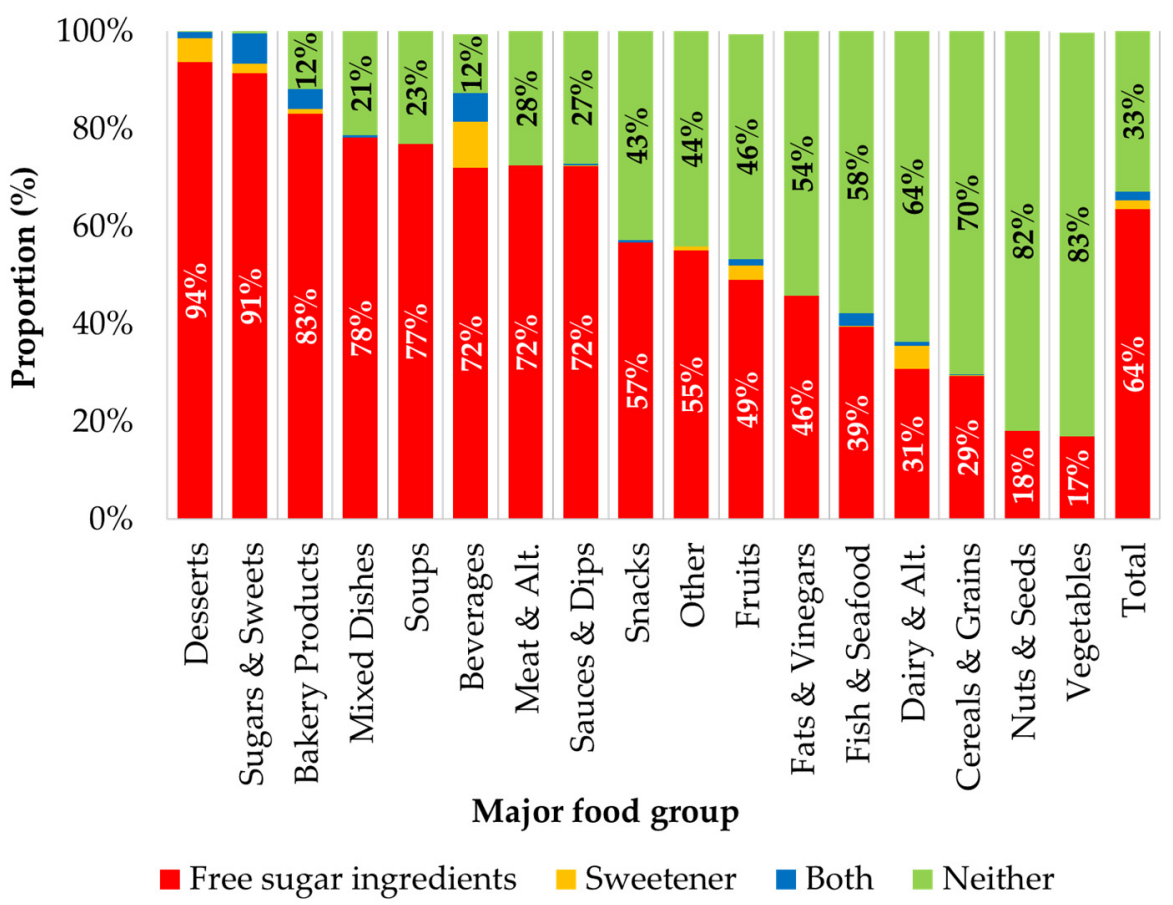

Figure 1. Proportion (\%) of prepackaged foods and beverages containing free sugar ingredients, sweeteners, a combination of both, or neither, by major food category and overall $(n=15,259)$. Proportions labelled on the figure only when value is $>10 \%$. "FSI" are those defined in Table 2. "Sweeteners" refers to all non- or low-caloric sweetening agents as defined by the Canadian Food Inspection Agency, including sugar alcohols (e.g., xylitol, and sorbitol), and non-caloric or artificial sweeteners (e.g., sucralose, and aspartame) [20]. Abbreviations: Alt. = Alternatives.

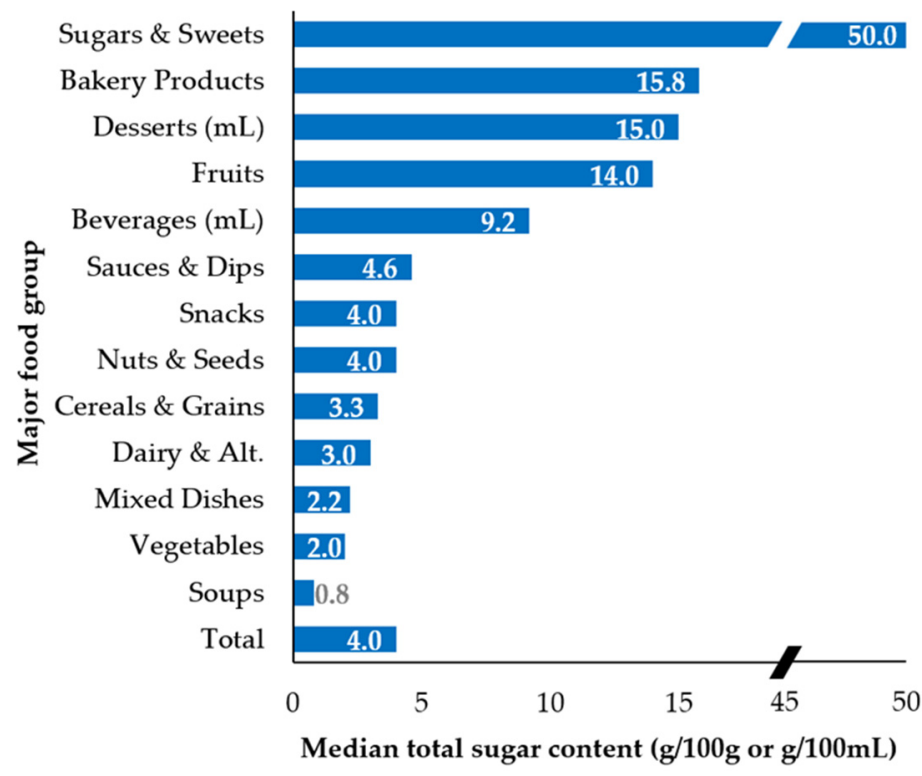

Figure 2. Cont. 


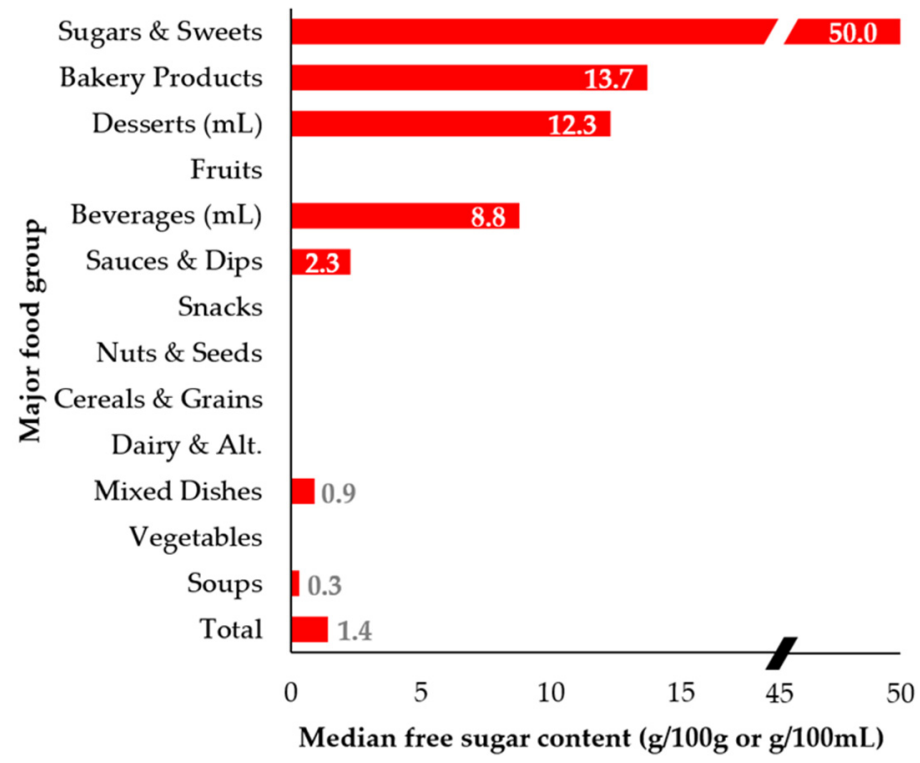

Figure 2. Median total sugar and free sugar content ( $\mathrm{g} / 100 \mathrm{~g}$ or $\mathrm{g} / 100 \mathrm{~mL}$ ) by major food group and overall ( $n=15,259)$ : (Top) median total sugar content; and (Bottom) median free sugar content. Categories with $0 \mathrm{~g} / 100 \mathrm{~g}$ or $100 \mathrm{~mL}$ median total sugar and free sugar (i.e., other foods and beverages; fats, oils, and vinegars; meat and alternatives; and fish and seafood) are not shown. ( $\boldsymbol{\bullet})$ denotes a break in the $\mathrm{x}$-axis between 20 and $45 \mathrm{~g} / 100 \mathrm{~g}$.

Table 2. Types of free sugar ingredients (FSI) identified in the FLIP 2013 database of Canadian prepackaged foods and beverages, by descending order of use $(n=15,259)$.

\begin{tabular}{|c|c|c|}
\hline Type & Examples $^{1}$ & $\begin{array}{l}n \text { (\% Foods } \\
\text { with FSI) }{ }^{2}\end{array}$ \\
\hline $\begin{array}{c}\text { Sugar (sucrose), } \\
\text { dried and granulated }\end{array}$ & $\begin{array}{l}\text { sugar, sucrose, brown sugar, cane sugar, pure sugar cane, pure cane sugar, raw } \\
\text { cane sugar, powdered sugar, golden sugar, golden cane sugar, granulated cane } \\
\text { sugar, granulated sugar cane juice, beet sugar, refined cane sugar, icing sugar, } \\
\text { dried sugar cane juice, demerara sugar, light brown sugar, refinery syrup powder, } \\
\text { invert sugar, evaporated cane juice, evaporated cane juice crystals, evaporated } \\
\text { milled sugar, milled cane sugar, evaporated sugar cane juice, caster sugar, coarse } \\
\text { sugar, turbinado sugar, natural cane sugar, turbinado cane sugar, white sugar, } \\
\text { whole cane sugar, yellow sugar, dehydrated cane juice, dehydrated cane sugar, } \\
\text { natural evaporated cane juice, confectioner's sugar, fondant sugar, raw sugar, } \\
\text { evaporated cane sugar, dehydrated cane syrup, dark brown sugar }\end{array}$ & $7517(49.3 \%)$ \\
\hline Glucose & $\begin{array}{l}\text { glucose, glucose solids, glucose syrup, dextrose, dextrose anhydrous, dextrose } \\
\text { syrup, anhydrous dextrose, dried glucose syrup, dextrin syrup }\end{array}$ & $2939(19.3 \%)$ \\
\hline Corn syrup & $\begin{array}{l}\text { corn syrup, corn syrup powder, corn syrup solids, high maltose corn syrup, dried } \\
\text { corn syrup extract, glucose-fructose, caramelized glucose-fructose, corn malt } \\
\text { syrup, fructose- glucose, glucose-fructose syrup, corn sweetener }\end{array}$ & $1626(10.7 \%)$ \\
\hline Fruit juice & $\begin{array}{l}\text { concentrated fruit juice, fruit juice, fruit juice concentrates, fruit juice } \\
\text { from concentrate }\end{array}$ & $1202(7.9 \%)$ \\
\hline High-fructose corn syrup ${ }^{3}$ & $\begin{array}{l}\text { high fructose corn syrup, sugar/glucose-fructose, sugar/fructose-glucose, sugar } \\
\text { and/or glucose-fructose, sugar and/or fructose-glucose }\end{array}$ & $873(5.7 \%)$ \\
\hline Molasses & $\begin{array}{l}\text { molasses, dehydrated molasses, powdered refiner's molasses, black molasses, } \\
\text { blackstrap molasses, dried molasses, refiner's molasses, cane juice molasses, dry } \\
\text { blackstrap molasses, dry molasses, fancy molasses, fancy molasses powder, } \\
\text { cooking molasses, molasses granules, molasses powder, molasses solids }\end{array}$ & $706(4.6 \%)$ \\
\hline Honey & $\begin{array}{l}\text { honey, liquid honey, amber honey, pasteurized honey, honey granules, honey } \\
\text { powder, honey solids, creamed honey, dried honey, granulated honey, raw honey, } \\
\text { buckwheat honey, dried honey powder, dry honey, white honey }\end{array}$ & $625(4.1 \%)$ \\
\hline Sugar (sucrose) syrups ${ }^{4}$ & $\begin{array}{l}\text { cane sugar syrup, sucrose syrup, dried cane syrup, cane syrup, cane refiner's } \\
\text { syrup, refined sugar syrup, burnt sugar syrup, invert cane syrup, golden syrup, } \\
\text { refiner's syrup, invert sugar syrup, caramel, caramel sugar syrup, caramel syrup, } \\
\text { evaporated cane syrup, liquid invert sugar, liquid sugar, liquid sucrose, } \\
\text { evaporated cane juice syrup, sugar cane syrup, treacle }\end{array}$ & $514(3.4 \%)$ \\
\hline
\end{tabular}


Table 2. Cont.

\begin{tabular}{|c|c|c|}
\hline Type & Examples $^{1}$ & $\begin{array}{l}n \text { ( } \% \text { Foods }^{2} \\
\text { with FSI) }{ }^{2}\end{array}$ \\
\hline Other syrups & $\begin{array}{l}\text { brown rice syrup, apple cider syrup, apple syrup, rice syrup, malt syrup, barley } \\
\text { malt syrup, malted barley syrup, tapioca syrup, raisin syrup, sorghum syrup, } \\
\text { wheat syrup }\end{array}$ & $439(2.9 \%)$ \\
\hline Fructose & fructose, fructose solids, fructose syrup, crystalline fructose & $324(2.1 \%)$ \\
\hline Other sugars & $\begin{array}{l}\text { potato syrup solids, palm sugar, tapioca sugar, tapioca syrup solids, lactose, } \\
\text { coconut sugar, oat syrup solids, maltose, isomaltose }\end{array}$ & $272(1.8 \%)$ \\
\hline Maple syrup & maple syrup, dehydrated maple syrup, maple sugar & $72(0.5 \%)$ \\
\hline Agave & agave, agave nectar & $27(0.2 \%)$ \\
\hline
\end{tabular}

${ }^{1} 152$ unique FSI were identified, not including different spellings, "organic" variations of nomenclature (e.g., organic cane sugar), claims of origin (e.g., Canadian maple syrup), claims of purity (e.g., 100\% pure agave) and specific flavours of fruit juice (e.g., apple juice, pear juice), are presented in descending order of use; determined from the number of products that contained each FSI; ${ }^{2}$ Combined percentage of foods containing a FSI exceeds $100 \%$ because 4642 (30.4\%) of the food supply or $46.6 \%$ of the products with a FSI contained more than 1 type of FSI; ${ }^{3}$ Labelling terminology used in Canada for high-fructose corn syrup; ${ }^{4}$ Caramel used for colour, when indicated within the ingredient list, was not considered a FSI. Abbreviations: FLIP = Food Label Information Program.

\subsection{Median Total and Free Sugar Content}

Median free sugar content overall was $1.4 \mathrm{~g}$ per $100 \mathrm{~g}$ (or $100 \mathrm{~mL}$ ), about one-third of the median total sugar content $(4.0 \mathrm{~g}$ per $100 \mathrm{~g} / \mathrm{mL}$ ) (Figure 2). Fruits had the fourth highest median total sugar content $(14.0 \mathrm{~g} / 100 \mathrm{~g})$ but was among the lowest free sugar containing food groups with $0 \mathrm{~g} / 100 \mathrm{~g}$. This was followed by beverages with a median $9.2 \mathrm{~g} / 100 \mathrm{~mL}$ total sugar and $8.8 \mathrm{~g} / 100 \mathrm{~mL}$ free sugar. All other food groups contained about half or less than these total and free sugar levels. For free sugar, this drop was even more dramatic, with all other categories containing less than $2.5 \mathrm{~g} / 100 \mathrm{~g}$. When examining the food supply in detail by subcategories (Table 3), the top total sugar containing subcategories were sugar (100 g/100 g), fruit snacks (72 g/100 g), dried fruits (55 g/100 g), dessert toppings and fillings $(53 \mathrm{~g} / 100 \mathrm{~mL})$, confectionery $(51 \mathrm{~g} / 100 \mathrm{~g})$, and sweet condiments $(50 \mathrm{~g} / 100 \mathrm{~g})$. The top free sugar containing subcategories were also sugar $(100 \mathrm{~g} / 100 \mathrm{~g})$, dessert toppings and fillings (53 g/100 mL), confectionery $(51 \mathrm{~g} / 100 \mathrm{~g})$, and sweet condiments $(50 \mathrm{~g} / 100 \mathrm{~g})$, however, dried fruits and fruit snacks were not among the top free sugar subcategories with $0 \mathrm{~g} / 100 \mathrm{~g}$ median free sugar content.

\subsection{Free Sugar as a Percent of Total Sugar}

Overall, free sugar accounted for $62 \%$ of the total sugar in prepackaged foods and beverages; the remainder was from naturally-occurring sources of sugar (Figure 3). In nearly half of the major food categories examined, free sugar contributed at least $75 \%$ of total sugar. This ranged from $100 \%$ of the total sugar in the food group sugars and sweets, to $11 \%$ of the total sugar in the nuts and seeds food group. Of the top sugar-dense food groups, free sugar as a proportion of total sugar for bakery products was $79 \%$, for desserts $81 \%$, and for beverages $86 \%$. Because some food categories contain very little total sugar, the addition of small amounts of free sugar can result in the percentages appearing quite high. For example, free sugar as a proportion of total sugar in fats and vinegars (81\%), other foods and beverages $(87 \%)$, and fish and seafood $(85 \%)$ are high, but all had a median free sugar content of $0 \mathrm{~g} / 100 \mathrm{~g}$. A more detailed evaluation of free sugar at the subcategory and minor category level (Table 3), revealed that free sugar accounted for $100 \%$ of the total sugar in cookies, energy drinks, fruit drinks, soft drinks, sports drinks, dessert toppings and fillings, mayonnaise, bacon, eggs, and all subcategories of the sugars and sweets food group. Additionally, free sugar accounted for $>90 \%$ of the total sugar in many baked desserts, muffins, cakes, cereal and granola bars, ready-to-eat cereals, and several minor categories of condiments and sauces. 
Table 3. Total and free sugar contents (g per $100 \mathrm{~g}$ or $100 \mathrm{~mL}$ ) and average free sugar as a proportion of total sugar (\%) in FLIP 2013 by food group, subcategory, and minor category $(n=15,259){ }^{1}$.

\begin{tabular}{|c|c|c|c|c|c|c|c|c|c|c|c|c|c|c|}
\hline \multirow{2}{*}{$\begin{array}{l}\text { Food Group, Subcategory, and } \\
\text { Minor Category }\end{array}$} & \multirow{2}{*}{$n$} & \multicolumn{6}{|c|}{ Total Sugar (g/100 g or $100 \mathrm{~mL})$} & \multicolumn{6}{|c|}{ Free Sugar (g/100 g or $100 \mathrm{~mL}$ ) } & \multirow{2}{*}{$\begin{array}{l}\text { Free Sugar as a } \\
\text { Percent of Tota } \\
\text { Sugar }^{2}\end{array}$} \\
\hline & & $\bar{X}$ (SD) & Min & 25th & 50th & 75th & Max & $\bar{X}(\mathrm{SD})$ & Min & 25th & 50th & 75th & Max & \\
\hline Bakery Products & 2197 & $17(15)$ & 0 & 4 & 16 & 29 & 94 & $16(14)$ & 0 & 2 & 14 & 28 & 94 & $79 \%$ \\
\hline Baked Breakfast & 123 & $10(7)$ & 1 & 5 & 7 & 10 & 38 & $5(8)$ & 0 & 0 & 2 & 5 & 34 & $29 \%$ \\
\hline Croissants & 6 & $11(5)$ & 6 & 7 & 8 & 17 & 18 & $5(6)$ & 1 & 1 & 2 & 12 & 12 & $38 \%$ \\
\hline Pancakes, Waffles, French Toast & 92 & $7(3)$ & 1 & 5 & 6 & 9 & 24 & $2(3)$ & 0 & 0 & 0 & 3 & 20 & $18 \%$ \\
\hline Tea Biscuits and Scones & 14 & $12(8)$ & 4 & 7 & 11 & 14 & 28 & $9(8)$ & 0 & 3 & 7 & 11 & 25 & $59 \%$ \\
\hline Toaster Pastries & 11 & $26(9)$ & 15 & 17 & 24 & 36 & 38 & $22(10)$ & 10 & 12 & 19 & 32 & 34 & $80 \%$ \\
\hline Baked Desserts & 88 & $30(11)$ & 8 & 22 & 28 & 39 & 50 & $27(10)$ & 6 & 18 & 24 & 36 & 46 & $88 \%$ \\
\hline Brownies/Squares & 39 & $39(6)$ & 22 & 35 & 40 & 43 & 50 & $36(7)$ & 18 & 33 & 36 & 40 & 46 & $92 \%$ \\
\hline Doughnut, Cake & 12 & $23(6)$ & 12 & 19 & 23 & 27 & 30 & $21(5)$ & 11 & 17 & 20 & 24 & 29 & $92 \%$ \\
\hline Doughnut, Yeast & 5 & $19(4)$ & 15 & 17 & 17 & 20 & 24 & $17(3)$ & 14 & 16 & 16 & 18 & 23 & $93 \%$ \\
\hline Fruit-Filled Pastries & 8 & $24(4)$ & 19 & 20 & 24 & 28 & 31 & $17(3)$ & 13 & 14 & 17 & 19 & 21 & $69 \%$ \\
\hline Other Pastries (e.g., eclairs) & 14 & $23(11)$ & 8 & 14 & 21 & 27 & 50 & $19(10)$ & 6 & 11 & 18 & 20 & 41 & $80 \%$ \\
\hline Sweet Buns (e.g., cinnamon rolls) & 10 & $23(6)$ & 11 & 22 & 25 & 27 & 32 & $21(6)$ & 9 & 21 & 23 & 25 & 29 & $91 \%$ \\
\hline Bread Products & 548 & $6(7)$ & 0 & 2 & 3 & 6 & 36 & $4(7)$ & 0 & 0 & 2 & 4 & 35 & $58 \%$ \\
\hline Bagels & 37 & $5(3)$ & 2 & 4 & 5 & 7 & 16 & $3(3)$ & 0 & 1 & 3 & 5 & 15 & $62 \%$ \\
\hline Bread w/ Additions (e.g., garlic bread) & 31 & $2(3)$ & 0 & 0 & 2 & 3 & 14 & $1(1)$ & 0 & 0 & 0 & 1 & 4 & $27 \%$ \\
\hline Bread w/ Raisins & 15 & $16(6)$ & 5 & 9 & 16 & 18 & 24 & $12(8)$ & 0 & 4 & 14 & 17 & 23 & $72 \%$ \\
\hline Diet Bread & 6 & $4(1)$ & 2 & 2 & 4 & 5 & 5 & $2(1)$ & 1 & 1 & 2 & 3 & 3 & $52 \%$ \\
\hline English Muffins & 24 & $2(2)$ & 0 & 2 & 2 & 2 & 13 & $0(1)$ & 0 & 0 & 0 & 0 & 2 & $19 \%$ \\
\hline Flatbreads (e.g., pita, naan, tortillas) & 131 & $3(4)$ & 0 & 0 & 2 & 4 & 20 & $2(4)$ & 0 & 0 & 1 & 3 & 19 & $60 \%$ \\
\hline Hearth Bread & 69 & $2(2)$ & 0 & 0 & 2 & 2 & 9 & $1(2)$ & 0 & 0 & 0 & 1 & 9 & $39 \%$ \\
\hline Muffins and Quick Breads & 57 & $24(7)$ & 7 & 20 & 25 & 28 & 36 & $22(7)$ & 0 & 19 & 23 & 27 & 35 & $93 \%$ \\
\hline Pantry Bread and Rolls & 178 & $4(2)$ & 0 & 3 & 4 & 5 & 20 & $2(2)$ & 0 & 1 & 2 & 3 & 8 & $56 \%$ \\
\hline Cake & 246 & $30(9)$ & 13 & 23 & 28 & 36 & 56 & $27(8)$ & 10 & 20 & 25 & 32 & 52 & $89 \%$ \\
\hline Cake Mixes & 52 & $23(5)$ & 16 & 20 & 22 & 24 & 36 & $22(5)$ & 15 & 19 & 20 & 23 & 36 & $95 \%$ \\
\hline Cakes w/Icing/Filling & 21 & $32(7)$ & 13 & 29 & 34 & 36 & 38 & $27(6)$ & 10 & 24 & 28 & 31 & 36 & $86 \%$ \\
\hline Cheesecakes & 35 & $25(4)$ & 18 & 22 & 25 & 27 & 32 & $21(3)$ & 15 & 18 & 21 & 23 & 27 & $83 \%$ \\
\hline Coffee Cakes w/o Icing/Filling & 42 & $28(5)$ & 19 & 25 & 27 & 30 & 43 & $26(4)$ & 18 & 23 & 26 & 28 & 41 & $95 \%$ \\
\hline Cream, Custard and Mousse Cake & 13 & $22(4)$ & 16 & 18 & 21 & 23 & 32 & $18(4)$ & 13 & 15 & 18 & 19 & 26 & $83 \%$ \\
\hline Cupcakes & 28 & $43(6)$ & 29 & 40 & 42 & 46 & 56 & $37(7)$ & 24 & 33 & 36 & 41 & 51 & $85 \%$ \\
\hline Ice Cream Cakes & 11 & $26(5)$ & 13 & 23 & 26 & 28 & 35 & $21(5)$ & 11 & 19 & 22 & 23 & 29 & $83 \%$ \\
\hline Sauce Cakes & 4 & $27(2)$ & 25 & 25 & 26 & 28 & 29 & $22(1)$ & 20 & 21 & 22 & 23 & 24 & $83 \%$ \\
\hline Snack Cakes & 29 & $41(8)$ & 24 & 36 & 43 & 46 & 54 & $37(8)$ & 23 & 31 & 37 & 44 & 52 & $91 \%$ \\
\hline Sponge Cakes w/o Icing/Filling & 6 & $36(4)$ & 30 & 34 & 37 & 38 & 43 & $36(4)$ & 30 & 33 & 37 & 37 & 42 & $99 \%$ \\
\hline Upside-down and Fruit Cakes & 5 & $31(2)$ & 28 & 30 & 31 & 32 & 34 & $26(2)$ & 23 & 25 & 26 & 27 & 28 & $83 \%$ \\
\hline Cereal/Granola Bars & 202 & 30 (7) & 11 & 24 & 31 & 35 & 57 & 27 (7) & 11 & 22 & 27 & 32 & 53 & $91 \%$ \\
\hline w/ Filling or Coating & 101 & $33(6)$ & 18 & 29 & 34 & 37 & 45 & $29(6)$ & 17 & 24 & 29 & 34 & 41 & $88 \%$ \\
\hline w/o Filling or Coating & 101 & $27(7)$ & 11 & 23 & 27 & 32 & 57 & $26(7)$ & 11 & 21 & 25 & 30 & 53 & $94 \%$ \\
\hline
\end{tabular}


Table 3. Cont.

\begin{tabular}{|c|c|c|c|c|c|c|c|c|c|c|c|c|c|c|}
\hline \multirow{2}{*}{$\begin{array}{l}\text { Food Group, Subcategory, and } \\
\text { Minor Category }\end{array}$} & \multirow{2}{*}{$n$} & \multicolumn{6}{|c|}{ Total Sugar (g/100 g or $100 \mathrm{~mL})$} & \multicolumn{6}{|c|}{ Free Sugar (g/100 g or $100 \mathrm{~mL})$} & \multirow{2}{*}{$\begin{array}{l}\text { Free Sugar as a } \\
\text { Percent of Total } \\
\text { Sugar }{ }^{2}\end{array}$} \\
\hline & & $\bar{X}(\mathrm{SD})$ & Min & 25th & 50th & 75th & Max & $\bar{X}(\mathrm{SD})$ & Min & 25th & 50th & 75th & Max & \\
\hline Cookies & 412 & $32(10)$ & 0 & 27 & 32 & 38 & 94 & $32(10)$ & 0 & 27 & 32 & 38 & 94 & $100 \%$ \\
\hline Chocolate Chip & 81 & $32(7)$ & 0 & 30 & 33 & 36 & 43 & $32(7)$ & 0 & 30 & 33 & 36 & 43 & $100 \%$ \\
\hline Chocolate Covered & 39 & 35 (11) & 0 & 29 & 37 & 41 & 56 & 35 (11) & 0 & 29 & 37 & 41 & 56 & $100 \%$ \\
\hline Fruit-Filled & 21 & $33(5)$ & 20 & 31 & 33 & 34 & 43 & $33(5)$ & 20 & 31 & 33 & 34 & 43 & $100 \%$ \\
\hline Other Cookies (e.g., macaroons, biscotti) & 116 & $31(11)$ & 0 & 26 & 30 & 36 & 94 & $31(11)$ & 0 & 26 & 30 & 36 & 94 & $100 \%$ \\
\hline Sandwich Cookies & 64 & $35(7)$ & 0 & 32 & 35 & 40 & 53 & $35(7)$ & 0 & 32 & 35 & 40 & 53 & $100 \%$ \\
\hline Shortbread & 8 & $20(10)$ & 0 & 16 & 22 & 27 & 30 & $20(10)$ & 0 & 16 & 22 & 27 & 30 & $100 \%$ \\
\hline Social Tea/Sugar-Type & 53 & $24(8)$ & 0 & 19 & 23 & 29 & 43 & $24(8)$ & 0 & 19 & 23 & 29 & 43 & $100 \%$ \\
\hline Sugar Wafer & 30 & 35 (17) & 0 & 26 & 40 & 47 & 58 & 35 (17) & 0 & 26 & 40 & 47 & 58 & $100 \%$ \\
\hline Dough and Pastry & 62 & $6(8)$ & 0 & 0 & 4 & 7 & 29 & $5(8)$ & 0 & 0 & 3 & 5 & 28 & $78 \%$ \\
\hline Pie Dough and Shells & 49 & $6(8)$ & 0 & 0 & 4 & 8 & 29 & $6(8)$ & 0 & 0 & 2 & 6 & 28 & $79 \%$ \\
\hline Pizza Crust & 13 & $3(1)$ & 2 & 3 & 4 & 4 & 6 & $3(1)$ & 1 & 2 & 3 & 3 & 5 & $77 \%$ \\
\hline Other Bakery Products & 416 & $6(6)$ & 0 & 0 & 5 & 8 & 30 & $5(6)$ & 0 & 0 & 4 & 7 & 30 & $85 \%$ \\
\hline Pies, Tarts, Cobblers, Crisps & 100 & $20(8)$ & 2 & 16 & 20 & 26 & 42 & $17(8)$ & 0 & 11 & 16 & 22 & 40 & $75 \%$ \\
\hline Butter/Sugar & 28 & $27(5)$ & 18 & 25 & 27 & 29 & 42 & $23(5)$ & 15 & 21 & 24 & 26 & 40 & $86 \%$ \\
\hline Custard-Based & 13 & $27(7)$ & 14 & 23 & 26 & 32 & 36 & $23(7)$ & 9 & 19 & 23 & 29 & 33 & $86 \%$ \\
\hline Fruit-Filled Pies & 59 & $16(6)$ & 2 & 14 & 16 & 18 & 36 & $12(6)$ & 0 & 10 & 12 & 14 & 33 & $67 \%$ \\
\hline Beverages $^{3}$ & 1407 & $8(5)$ & 0 & 4 & 9 & 11 & 17 & $7(5)$ & 0 & 1 & 9 & 11 & 17 & $86 \%$ \\
\hline Dairy and Alternatives & 242 & $6(4)$ & 0 & 3 & 5 & 10 & 15 & $3(3)$ & 0 & 0 & 3 & 6 & 12 & $49 \%$ \\
\hline Drinkable Yogurt & 35 & $11(3)$ & 3 & 11 & 12 & 13 & 14 & $7(3)$ & 0 & 7 & 8 & 10 & 11 & $63 \%$ \\
\hline Milk, Flavoured & 28 & $10(2)$ & 5 & 9 & 10 & 11 & 12 & $5(2)$ & 0 & 4 & 6 & 7 & 8 & $52 \%$ \\
\hline Milk, Plain & 52 & $5(0)$ & 3 & 4 & 4 & 5 & 6 & $0(0)$ & 0 & 0 & 0 & 0 & 0 & $0 \%$ \\
\hline Plant-Based Milk, Flavoured & 55 & $5(2)$ & 0 & 4 & 5 & 7 & 10 & $4(3)$ & 0 & 3 & 4 & 7 & 9 & $79 \%$ \\
\hline Plant-Based Milk, Plain & 59 & $2(1)$ & 0 & 0 & 2 & 3 & 5 & $1(1)$ & 0 & 0 & 1 & 2 & 5 & $54 \%$ \\
\hline Shakes & 11 & $12(4)$ & 6 & 6 & 14 & 14 & 15 & $9(4)$ & 3 & 3 & 11 & 11 & 12 & $70 \%$ \\
\hline Smoothies & 2 & $8(2)$ & 7 & 7 & 8 & 10 & 10 & $5(2)$ & 4 & 4 & 5 & 6 & 6 & $60 \%$ \\
\hline Energy Drinks & 14 & $7(5)$ & 0 & 0 & 7 & 11 & 14 & $7(5)$ & 0 & 0 & 7 & 11 & 14 & $100 \%$ \\
\hline Energy Drinks, Diet or Light & 7 & $2(2)$ & 0 & 0 & 0 & 4 & 4 & $2(2)$ & 0 & 0 & 0 & 4 & 4 & $100 \%$ \\
\hline Energy Drinks, Regular & 7 & $12(1)$ & 11 & 11 & 11 & 13 & 14 & $12(1)$ & 11 & 11 & 11 & 13 & 14 & $100 \%$ \\
\hline Fruit Drinks & 654 & $10(3)$ & 0 & 9 & 10 & 12 & 17 & $10(3)$ & 0 & 9 & 10 & 12 & 17 & $100 \%$ \\
\hline Fruit Drink & 40 & $9(2)$ & 3 & 10 & 10 & 10 & 12 & $9(2)$ & 3 & 10 & 10 & 10 & 12 & $100 \%$ \\
\hline Fruit Juice & 378 & $10(3)$ & 0 & 9 & 10 & 12 & 17 & $10(3)$ & 0 & 9 & 10 & 12 & 17 & $100 \%$ \\
\hline Fruit Juice-Drink, Combination & 236 & $10(3)$ & 0 & 9 & 11 & 12 & 17 & $10(3)$ & 0 & 9 & 11 & 12 & 17 & $100 \%$ \\
\hline Hot Beverages & 58 & $7(4)$ & 0 & 4 & 9 & 11 & 14 & $6(4)$ & 0 & 3 & 7 & 9 & 13 & $83 \%$ \\
\hline Cocoa o & 27 & $9(3)$ & 2 & 8 & 10 & 11 & 14 & $7(4)$ & 0 & 6 & 9 & 9 & 12 & $70 \%$ \\
\hline Coffee, Flavoured/Sweetened & 27 & $5(4)$ & 0 & 1 & 5 & 9 & 13 & $5(4)$ & 0 & 1 & 5 & 9 & 13 & $100 \%$ \\
\hline Tea, Sweetened & 4 & $6(4)$ & 1 & 3 & 6 & 9 & 9 & $4(3)$ & 0 & 2 & 5 & 7 & 7 & $65 \%$ \\
\hline Other Beverages & 39 & $1(3)$ & 0 & 0 & 0 & 0 & 15 & $1(3)$ & 0 & 0 & 0 & 0 & 15 & $67 \%$ \\
\hline Soft Drinks & 272 & $7(5)$ & 0 & 0 & 9 & 11 & 16 & $7(5)$ & 0 & 0 & 9 & 11 & 16 & $100 \%$ \\
\hline
\end{tabular}


Table 3. Cont

\begin{tabular}{|c|c|c|c|c|c|c|c|c|c|c|c|c|c|c|}
\hline \multirow{2}{*}{$\begin{array}{l}\text { Food Group, Subcategory, and } \\
\text { Minor Category }\end{array}$} & \multirow{2}{*}{$n$} & \multicolumn{6}{|c|}{ Total Sugar (g/100 g or $100 \mathrm{~mL})$} & \multicolumn{6}{|c|}{ Free Sugar (g/100 g or 100 mL) } & \multirow{2}{*}{$\begin{array}{l}\text { Free Sugar as a } \\
\text { Percent of Total } \\
\text { Sugar }^{2}\end{array}$} \\
\hline & & $\bar{X}(\mathrm{SD})$ & Min & 25th & 50th & 75th & Max & $\bar{X}(\mathrm{SD})$ & Min & 25th & 50th & 75th & Max & \\
\hline Iced Tea, Diet or Light & 13 & $1(1)$ & 0 & 0 & 0 & 0 & 4 & $1(1)$ & 0 & 0 & 0 & 0 & 4 & $100 \%$ \\
\hline Iced Tea, Regular & 52 & $8(2)$ & 0 & 7 & 9 & 10 & 12 & $8(2)$ & 0 & 7 & 9 & 10 & 12 & $100 \%$ \\
\hline Soft Drink, Regular & 121 & $11(2)$ & 5 & 10 & 11 & 13 & 16 & $11(2)$ & 5 & 10 & 11 & 13 & 16 & $100 \%$ \\
\hline Soft Drink, Diet or Light & 86 & $0(0)$ & 0 & 0 & 0 & 0 & 0 & $0(0)$ & 0 & 0 & 0 & 0 & 0 & \\
\hline Sports Drinks & 30 & $4(2)$ & 0 & 2 & 6 & 6 & 6 & $4(2)$ & 0 & 2 & 6 & 6 & 6 & $100 \%$ \\
\hline Sports Drinks, Diet or Light & 11 & $1(1)$ & 0 & 0 & 2 & 2 & 3 & $1(1)$ & 0 & 0 & 2 & 2 & 3 & $100 \%$ \\
\hline Sports Drinks, Regular & 19 & $6(0)$ & 5 & 6 & 6 & 6 & 6 & $6(0)$ & 5 & 6 & 6 & 6 & 6 & $100 \%$ \\
\hline Vegetable Drinks & 43 & $3(1)$ & 1 & 2 & 3 & 4 & 6 & $1(1)$ & 0 & 0 & 0 & 1 & 4 & $16 \%$ \\
\hline Water & 55 & $0(0)$ & 0 & 0 & 0 & 0 & 0 & $0(0)$ & 0 & 0 & 0 & 0 & 0 & \\
\hline Cereals and Grain Products & 969 & $8(11)$ & 0 & 0 & 3 & 14 & 53 & $6(10)$ & 0 & 0 & 0 & 10 & 53 & $33 \%$ \\
\hline Hot Breakfast Cereal & 107 & $12(13)$ & 0 & 0 & 3 & 24 & 42 & $11(13)$ & 0 & 0 & 0 & 23 & 41 & $76 \%$ \\
\hline Flavoured/Sweetened & 48 & $26(7)$ & 9 & 21 & 25 & 29 & 42 & $25(8)$ & 0 & 20 & 25 & 29 & 41 & $96 \%$ \\
\hline Plain & 59 & $1(2)$ & 0 & 0 & 0 & 0 & 10 & $0(0)$ & 0 & 0 & 0 & 0 & 0 & $0 \%$ \\
\hline Other Cereals and Grains & 612 & $2(2)$ & 0 & 0 & 2 & 4 & 29 & $0(1)$ & 0 & 0 & 0 & 0 & 27 & $0 \%$ \\
\hline Ready-to-Eat Breakfast Cereal & 250 & $21(10)$ & 0 & 16 & 20 & 26 & 53 & $17(10)$ & 0 & 11 & 17 & 22 & 53 & $76 \%$ \\
\hline Flakes & 36 & $18(10)$ & 7 & 10 & 13 & 23 & 43 & $13(11)$ & 1 & 5 & 8 & 18 & 40 & $60 \%$ \\
\hline Flakes w/ Fruit and/or Nuts & 30 & $25(6)$ & 16 & 22 & 24 & 28 & 42 & $21(6)$ & 11 & 17 & 19 & 23 & 38 & $81 \%$ \\
\hline Granola/Muesli & 84 & $21(5)$ & 4 & 18 & 22 & 25 & 32 & $16(6)$ & 0 & 13 & 17 & 21 & 28 & $72 \%$ \\
\hline High-Fibre Compact & 8 & $16(8)$ & 0 & 13 & 18 & 20 & 25 & $16(8)$ & 0 & 13 & 18 & 20 & 25 & $100 \%$ \\
\hline Puffed & 13 & $15(17)$ & 0 & 3 & 10 & 22 & 53 & $15(17)$ & 0 & 3 & 10 & 22 & 53 & $100 \%$ \\
\hline Semi-Compact/Formed & 59 & $25(12)$ & 3 & 16 & 20 & 34 & 53 & $21(13)$ & 0 & 11 & 15 & 31 & 51 & $74 \%$ \\
\hline Shredded & 20 & $16(8)$ & 0 & 15 & 18 & 22 & 24 & $16(8)$ & 0 & 15 & 18 & 22 & 24 & $100 \%$ \\
\hline Dairy Products and Substitutes & 1003 & $6(9)$ & 0 & 0 & 3 & 10 & 77 & $3(8)$ & 0 & 0 & 0 & 5 & 76 & $41 \%$ \\
\hline Cottage Cheese & 26 & $5(2)$ & 3 & 4 & 5 & 5 & 12 & $1(2)$ & 0 & 0 & 0 & 0 & 8 & $12 \%$ \\
\hline Cottage Cheese, Flavoured & 5 & $10(2)$ & 8 & 8 & 10 & 12 & 12 & $6(2)$ & 4 & 4 & 6 & 8 & 8 & $57 \%$ \\
\hline Cottage Cheese, Plain & 21 & $4(1)$ & 3 & 4 & 4 & 5 & 6 & $0(0)$ & 0 & 0 & 0 & 0 & 1 & $2 \%$ \\
\hline Cream or Cream Substitute & 85 & $14(13)$ & 0 & 6 & 11 & 22 & 58 & $13(13)$ & 0 & 0 & 10 & 22 & 57 & $73 \%$ \\
\hline Cream, Aerosol or Whipped & 27 & $16(5)$ & 7 & 11 & 17 & 22 & 25 & $15(6)$ & 4 & 11 & 15 & 20 & 23 & $90 \%$ \\
\hline Cream, Liquid & 50 & $15(15)$ & 0 & 6 & 7 & 33 & 58 & $13(16)$ & 0 & 0 & 3 & 33 & 57 & $61 \%$ \\
\hline Cream, Powder & 8 & $0(0)$ & 0 & 0 & 0 & 0 & 0 & $0(0)$ & 0 & 0 & 0 & 0 & 0 & \\
\hline Cream Cheese & 65 & $7(4)$ & 0 & 7 & 7 & 7 & 25 & $1(4)$ & 0 & 0 & 0 & 0 & 20 & $9 \%$ \\
\hline Cream Cheese, Flavoured & 37 & $8(4)$ & 0 & 7 & 7 & 7 & 25 & $2(5)$ & 0 & 0 & 0 & 1 & 20 & $15 \%$ \\
\hline Cream Cheese, Plain & 28 & $5(2)$ & 0 & 3 & 7 & 7 & 10 & $0(0)$ & 0 & 0 & 0 & 0 & 0 & $0 \%$ \\
\hline Milk, Condensed & 36 & $18(25)$ & 0 & 2 & 6 & 37 & 77 & $15(26)$ & 0 & 0 & 0 & 30 & 76 & $29 \%$ \\
\hline Coconut Milk (canned) & 18 & $7(18)$ & 0 & 1 & 2 & 2 & 77 & $5(18)$ & 0 & 0 & 0 & 0 & 76 & $11 \%$ \\
\hline Condensed Milk & 8 & $58(2)$ & 57 & 57 & 57 & 61 & 62 & $57(2)$ & 55 & 55 & 55 & 59 & 60 & $97 \%$ \\
\hline Evaporated Milk & 10 & $7(2)$ & 6 & 6 & 6 & 6 & 13 & $0(0)$ & 0 & 0 & 0 & 0 & 0 & $0 \%$ \\
\hline Other Dairy Products and Substitutes & 468 & $1(6)$ & 0 & 0 & 0 & 0 & 60 & $0(2)$ & 0 & 0 & 0 & 0 & 9 & $42 \%$ \\
\hline Cheese, unless listed separately & 327 & $0(3)$ & 0 & 0 & 0 & 0 & 50 & $0(0)$ & 0 & 0 & 0 & 0 & 0 & $0 \%$ \\
\hline Dairy-Free Cheese and Spreads & 13 & $1(1)$ & 0 & 0 & 0 & 0 & 4 & $0(0)$ & 0 & 0 & 0 & 0 & 0 & $0 \%$ \\
\hline
\end{tabular}


Table 3. Cont

\begin{tabular}{|c|c|c|c|c|c|c|c|c|c|c|c|c|c|c|}
\hline \multirow{2}{*}{$\begin{array}{l}\text { Food Group, Subcategory, and } \\
\text { Minor Category }\end{array}$} & \multirow{2}{*}{$n$} & \multicolumn{6}{|c|}{ Total Sugar (g/100 g or $100 \mathrm{~mL})$} & \multicolumn{6}{|c|}{ Free Sugar (g/100 g or $100 \mathrm{~mL}$ ) } & \multirow{2}{*}{$\begin{array}{l}\text { Free Sugar as a } \\
\text { Percent of Total } \\
\text { Sugar }{ }^{2}\end{array}$} \\
\hline & & $\bar{X}(\mathrm{SD})$ & Min & 25th & 50th & 75th & Max & $\bar{X}(\mathrm{SD})$ & Min & 25th & 50th & 75th & Max & \\
\hline Feta and Feta-Style Cheese & 40 & $0(0)$ & 0 & 0 & 0 & 0 & 0 & $0(0)$ & 0 & 0 & 0 & 0 & 0 & \\
\hline Hard Cheese & 32 & $7(18)$ & 0 & 0 & 0 & 0 & 60 & $0(0)$ & 0 & 0 & 0 & 0 & 0 & $0 \%$ \\
\hline Processed Cheese & 56 & $7(3)$ & 0 & 5 & 7 & 10 & 13 & $4(4)$ & 0 & 0 & 4 & 9 & 9 & $53 \%$ \\
\hline Soft Cheese & 71 & $3(5)$ & 0 & 0 & 2 & 4 & 18 & $2(4)$ & 0 & 0 & 0 & 1 & 17 & $40 \%$ \\
\hline Soft Cheese, Savoury/Plain & 11 & $4(1)$ & 3 & 3 & 4 & 4 & 5 & $0(0)$ & 0 & 0 & 0 & 0 & 0 & $0 \%$ \\
\hline Soft Cheese, Sweet & 45 & $1(1)$ & 0 & 0 & 0 & 0 & 4 & $0(0)$ & 0 & 0 & 0 & 0 & 2 & $13 \%$ \\
\hline Ricotta & 15 & $11(4)$ & 3 & 10 & 10 & 11 & 18 & $10(4)$ & 2 & 9 & 9 & 10 & 17 & $88 \%$ \\
\hline Sour Cream & 17 & $5(2)$ & 3 & 3 & 6 & 6 & 7 & $0(0)$ & 0 & 0 & 0 & 0 & 0 & $0 \%$ \\
\hline Yogurt & 235 & $9(4)$ & 1 & 4 & 11 & 12 & 17 & $6(4)$ & 0 & 0 & 8 & 9 & 14 & $48 \%$ \\
\hline Yogurt, Flavoured & 206 & $10(4)$ & 2 & 7 & 11 & 12 & 17 & $6(4)$ & 0 & 3 & 8 & 9 & 14 & $55 \%$ \\
\hline Yogurt, Plain & 29 & $3(1)$ & 1 & 3 & 3 & 4 & 6 & $0(0)$ & 0 & 0 & 0 & 0 & 0 & $0 \%$ \\
\hline Desserts 3,4 & 940 & $19(19)$ & 0 & 11 & 15 & 20 & 160 & $17(20)$ & 0 & 8 & 12 & 17 & 160 & $81 \%$ \\
\hline Custard, Gelatin, Mousse and Pudding & 195 & $13(7)$ & 0 & 10 & 15 & 18 & 26 & $12(7)$ & 0 & 9 & 14 & 16 & 26 & $86 \%$ \\
\hline Custard & 8 & $12(4)$ & 9 & 10 & 11 & 14 & 19 & $12(4)$ & 8 & 9 & 11 & 14 & 19 & $94 \%$ \\
\hline Gelatin & 80 & $12(8)$ & 0 & 0 & 15 & 15 & 26 & $12(8)$ & 0 & 0 & 15 & 15 & 26 & $100 \%$ \\
\hline Mousse & 6 & $5(5)$ & 0 & 3 & 4 & 10 & 12 & $5(4)$ & 0 & 2 & 3 & 9 & 11 & $83 \%$ \\
\hline Pudding & 101 & $15(6)$ & 0 & 12 & 16 & 19 & 22 & $12(6)$ & 0 & 11 & 14 & 16 & 21 & $78 \%$ \\
\hline Frozen Desserts & 626 & $14(5)$ & 0 & 11 & 14 & 17 & 31 & $11(5)$ & 0 & 7 & 10 & 14 & 30 & $75 \%$ \\
\hline Bars & 134 & $19(5)$ & 0 & 16 & 20 & 23 & 31 & $17(5)$ & 0 & 14 & 18 & 21 & 29 & $89 \%$ \\
\hline Cones, Filled & 25 & $16(4)$ & 3 & 15 & 16 & 19 & 20 & $14(4)$ & 0 & 12 & 13 & 16 & 17 & $79 \%$ \\
\hline Dairy-Free & 14 & $11(2)$ & 10 & 10 & 11 & 13 & 14 & $9(2)$ & 7 & 7 & 10 & 11 & 12 & $80 \%$ \\
\hline Frozen Yogurt & 56 & $12(2)$ & 9 & 11 & 12 & 13 & 24 & $9(2)$ & 6 & 8 & 9 & 10 & 21 & $75 \%$ \\
\hline Ice Cream, Ice Milk & 292 & $12(3)$ & 4 & 10 & 12 & 14 & 23 & $8(3)$ & 0 & 6 & 8 & 10 & 20 & $65 \%$ \\
\hline Ice Pops, Juice Bars, Cups & 37 & $17(6)$ & 0 & 15 & 17 & 20 & 30 & $17(6)$ & 0 & 15 & 17 & 20 & 30 & $100 \%$ \\
\hline Sandwiches & 34 & $13(3)$ & 4 & 12 & 13 & 15 & 20 & $9(3)$ & 0 & 8 & 9 & 11 & 17 & $69 \%$ \\
\hline Sherbet and Sorbet & 24 & $16(4)$ & 10 & 13 & 16 & 19 & 25 & $14(4)$ & 7 & 11 & 14 & 17 & 25 & $85 \%$ \\
\hline Sundaes & 10 & $14(3)$ & 10 & 12 & 15 & 17 & 18 & $11(3)$ & 7 & 9 & 12 & 14 & 16 & $79 \%$ \\
\hline Toppings and Fillings & 119 & $56(33)$ & 0 & 28 & 53 & 70 & 160 & $56(33)$ & 0 & 28 & 53 & 70 & 160 & $100 \%$ \\
\hline Cake Frostings and Icings & 60 & $71(35)$ & 0 & 46 & 65 & 100 & 160 & $71(35)$ & 0 & 46 & 65 & 100 & 160 & $100 \%$ \\
\hline Pie Fillings & 31 & $26(9)$ & 8 & 21 & 25 & 28 & 53 & $26(9)$ & 8 & 21 & 25 & 28 & 53 & $100 \%$ \\
\hline Toppings, Dips, Spreads & 28 & $57(22)$ & 0 & 50 & 55 & 69 & 113 & $57(22)$ & 0 & 50 & 55 & 69 & 113 & $100 \%$ \\
\hline Fats, Oils and Vinegars & 592 & $6(9)$ & 0 & 0 & 0 & 7 & 63 & $4(7)$ & 0 & 0 & 0 & 6 & 43 & $81 \%$ \\
\hline Butter, Margarine, Oils & 242 & $0(0)$ & 0 & 0 & 0 & 0 & 0 & $0(0)$ & 0 & 0 & 0 & 0 & 0 & \\
\hline Mayonnaise & 39 & $5(6)$ & 0 & 0 & 0 & 13 & 20 & $5(6)$ & 0 & 0 & 0 & 13 & 20 & $100 \%$ \\
\hline Salad Dressing & 311 & $10(11)$ & 0 & 0 & 7 & 19 & 63 & $7(8)$ & 0 & 0 & 5 & 12 & 43 & $79 \%$ \\
\hline Salad Dressings & 253 & $9(8)$ & 0 & 6 & 7 & 13 & 43 & $8(8)$ & 0 & 2 & 6 & 13 & 43 & $91 \%$ \\
\hline Vinegars & 58 & $16(17)$ & 0 & 0 & 13 & 31 & 63 & $2(7)$ & 0 & 0 & 0 & 0 & 42 & $12 \%$ \\
\hline Fish and Seafood & 434 & $1(2)$ & 0 & 0 & 0 & 1 & 22 & $1(2)$ & 0 & 0 & 0 & 1 & 22 & $85 \%$ \\
\hline Fruits & 444 & $25(23)$ & 0 & 9 & 14 & 38 & 83 & $8(15)$ & 0 & 0 & 0 & 9 & 73 & $30 \%$ \\
\hline Canned Fruit & 157 & $12(4)$ & 4 & 10 & 12 & 14 & 31 & $7(4)$ & 0 & 5 & 7 & 10 & 28 & $54 \%$ \\
\hline
\end{tabular}


Table 3. Cont

\begin{tabular}{|c|c|c|c|c|c|c|c|c|c|c|c|c|c|c|}
\hline \multirow{2}{*}{$\begin{array}{l}\text { Food Group, Subcategory, and } \\
\text { Minor Category }\end{array}$} & \multirow{2}{*}{$n$} & \multicolumn{6}{|c|}{ Total Sugar (g/100 g or $100 \mathrm{~mL})$} & \multicolumn{6}{|c|}{ Free Sugar (g/100 g or $100 \mathrm{~mL}$ ) } & \multirow{2}{*}{$\begin{array}{l}\text { Free Sugar as a } \\
\text { Percent of Total } \\
\text { Sugar }{ }^{2}\end{array}$} \\
\hline & & $\bar{X}(\mathrm{SD})$ & Min & 25th & 50th & 75th & Max & $\bar{X}(\mathrm{SD})$ & Min & 25th & 50th & 75th & Max & \\
\hline Canned in Juice & 70 & $12(3)$ & 5 & 9 & 12 & 14 & 16 & $7(3)$ & 0 & 4 & 7 & 9 & 11 & $53 \%$ \\
\hline Canned in Syrup & 73 & $13(3)$ & 8 & 11 & 13 & 15 & 31 & $9(3)$ & 4 & 7 & 8 & 11 & 28 & $66 \%$ \\
\hline Canned in Water & 14 & $5(1)$ & 4 & 4 & 5 & 6 & 8 & $0(0)$ & 0 & 0 & 0 & 0 & 0 & $0 \%$ \\
\hline Dried Fruit & 152 & $51(20)$ & 7 & 33 & 55 & 68 & 83 & $15(23)$ & 0 & 0 & 0 & 32 & 73 & $22 \%$ \\
\hline Sweetened Dried Fruit & 51 & $65(14)$ & 33 & 65 & 68 & 76 & 83 & $44(17)$ & 0 & 32 & 38 & 65 & 73 & $66 \%$ \\
\hline Unsweetened Dried Fruit & 101 & $43(18)$ & 7 & 33 & 38 & 60 & 75 & $0(0)$ & 0 & 0 & 0 & 0 & 0 & $0 \%$ \\
\hline Frozen Fruit & 61 & $8(3)$ & 4 & 6 & 7 & 9 & 15 & $0(0)$ & 0 & 0 & 0 & 0 & 0 & $0 \%$ \\
\hline Fruit Sauces & 62 & $12(3)$ & 7 & 9 & 10 & 15 & 20 & $3(3)$ & 0 & 0 & 0 & 5 & 11 & $16 \%$ \\
\hline Fruit Sauce, Sweetened & 27 & $15(2)$ & 13 & 14 & 15 & 16 & 20 & $6(2)$ & 0 & 5 & 5 & 7 & 11 & $38 \%$ \\
\hline Fruit Sauce, Unsweetened & 35 & $9(1)$ & 7 & 8 & 9 & 10 & 13 & $0(0)$ & 0 & 0 & 0 & 0 & 0 & $0 \%$ \\
\hline Other Fruits & 12 & $14(21)$ & 0 & 0 & 0 & 40 & 50 & $14(21)$ & 0 & 0 & 0 & 40 & 50 & $80 \%$ \\
\hline Fruit Garnish (e.g., maraschino cherries) & 4 & $43(5)$ & 40 & 40 & 40 & 45 & 50 & $42(5)$ & 40 & 40 & 40 & 45 & 50 & $100 \%$ \\
\hline Fruit Juice Ingredients & 8 & $0(0)$ & 0 & 0 & 0 & 0 & 1 & $0(0)$ & 0 & 0 & 0 & 0 & 0 & $0 \%$ \\
\hline Meat, Eggs and Substitutes & 959 & $2(3)$ & 0 & 0 & 0 & 2 & 21 & $1(3)$ & 0 & 0 & 0 & 2 & 21 & $80 \%$ \\
\hline Bacon oo & 58 & $0(1)$ & 0 & 0 & 0 & 0 & 4 & $0(1)$ & 0 & 0 & 0 & 0 & 4 & $100 \%$ \\
\hline Cooked & 20 & $0(1)$ & 0 & 0 & 0 & 0 & 4 & $0(1)$ & 0 & 0 & 0 & 0 & 4 & $100 \%$ \\
\hline Uncooked & 38 & $0(1)$ & 0 & 0 & 0 & 0 & 2 & $0(1)$ & 0 & 0 & 0 & 0 & 2 & $100 \%$ \\
\hline Deli Meats & 257 & $1(1)$ & 0 & 0 & 0 & 2 & 5 & $1(1)$ & 0 & 0 & 0 & 2 & 5 & $93 \%$ \\
\hline Dry-cured & 90 & $1(1)$ & 0 & 0 & 0 & 1 & 3 & $1(1)$ & 0 & 0 & 0 & 0 & 3 & $90 \%$ \\
\hline Fully Cooked & 167 & $1(1)$ & 0 & 0 & 1 & 2 & 5 & $1(1)$ & 0 & 0 & 0 & 2 & 5 & $94 \%$ \\
\hline Eggs and Egg Substitutes & 56 & $0(2)$ & 0 & 0 & 0 & 0 & 10 & $0(2)$ & 0 & 0 & 0 & 0 & 10 & $100 \%$ \\
\hline Meat and Poultry & 498 & $2(3)$ & 0 & 0 & 1 & 3 & 21 & $2(3)$ & 0 & 0 & 0 & 2 & 21 & $78 \%$ \\
\hline Meat Substitutes & 90 & $2(2)$ & 0 & 0 & 1 & 2 & 11 & $1(2)$ & 0 & 0 & 1 & 2 & 11 & $63 \%$ \\
\hline Meat Analogues & 74 & $2(2)$ & 0 & 0 & 1 & 2 & 11 & $1(2)$ & 0 & 0 & 1 & 2 & 11 & $66 \%$ \\
\hline Plain Tofu & 10 & $1(1)$ & 0 & 0 & 0 & 1 & 2 & $0(0)$ & 0 & 0 & 0 & 0 & 0 & $0 \%$ \\
\hline Seasoned Tofu and Tempeh & 3 & $4(4)$ & 0 & 0 & 4 & 9 & 9 & $4(4)$ & 0 & 0 & 4 & 9 & 9 & $100 \%$ \\
\hline Sweetened Tofu & 3 & $11(1)$ & 10 & 10 & 11 & 11 & 11 & $10(1)$ & 10 & 10 & 10 & 11 & 11 & $96 \%$ \\
\hline Mixed Dishes, Sides and Entrees & 1580 & $3(2)$ & 0 & 1 & 2 & 4 & 20 & $2(2)$ & 0 & 0 & 1 & 2 & 19 & $48 \%$ \\
\hline Beans & 36 & $6(4)$ & 0 & 1 & 7 & 8 & 12 & $5(4)$ & 0 & 0 & 6 & 8 & 12 & $79 \%$ \\
\hline Baked Beans & 26 & $8(2)$ & 4 & 6 & 8 & 9 & 12 & $7(2)$ & 4 & 6 & 8 & 8 & 12 & $95 \%$ \\
\hline Refried Beans & 10 & $1(0)$ & 0 & 0 & 1 & 1 & 1 & $0(0)$ & 0 & 0 & 0 & 0 & 0 & $17 \%$ \\
\hline Canned Chili & 21 & $2(1)$ & 1 & 2 & 2 & 3 & 4 & $1(1)$ & 0 & 0 & 1 & 1 & 2 & $22 \%$ \\
\hline Mixed Dishes, Other & 37 & $3(2)$ & 0 & 1 & 3 & 4 & 8 & $1(1)$ & 0 & 0 & 1 & 2 & 6 & $28 \%$ \\
\hline Other Mixed Dishes & 17 & $2(2)$ & 0 & 1 & 1 & 3 & 8 & $1(2)$ & 0 & 0 & 0 & 0 & 6 & $17 \%$ \\
\hline Taco Kits & 20 & $3(1)$ & 2 & 3 & 4 & 4 & 6 & $1(1)$ & 0 & 1 & 2 & 2 & 4 & $36 \%$ \\
\hline Pizza and Frozen Sandwiches & 214 & $3(2)$ & 1 & 2 & 3 & 4 & 10 & $3(2)$ & 0 & 1 & 2 & 3 & 9 & $72 \%$ \\
\hline Pizza & 161 & $3(2)$ & 1 & 2 & 3 & 4 & 8 & $3(2)$ & 0 & 1 & 2 & 4 & 7 & $71 \%$ \\
\hline Pizza Snacks and Sandwiches & 53 & $4(2)$ & 1 & 3 & 3 & 4 & 10 & $3(2)$ & 0 & 2 & 2 & 3 & 9 & $76 \%$ \\
\hline Potatoes & 126 & $1(2)$ & 0 & 0 & 1 & 2 & 9 & $0(1)$ & 0 & 0 & 0 & 0 & 8 & $20 \%$ \\
\hline
\end{tabular}


Table 3. Cont

\begin{tabular}{|c|c|c|c|c|c|c|c|c|c|c|c|c|c|c|}
\hline \multirow{2}{*}{$\begin{array}{l}\text { Food Group, Subcategory, and } \\
\text { Minor Category }\end{array}$} & \multirow{2}{*}{$n$} & \multicolumn{6}{|c|}{ Total Sugar (g/100 g or $100 \mathrm{~mL})$} & \multicolumn{6}{|c|}{ Free Sugar (g/100 g or $100 \mathrm{~mL}$ ) } & \multirow{2}{*}{$\begin{array}{l}\text { Free Sugar as a } \\
\text { Percent of Total } \\
\text { Sugar }^{2}\end{array}$} \\
\hline & & $\bar{X}(\mathrm{SD})$ & Min & 25th & 50th & 75th & Max & $\bar{X}(\mathrm{SD})$ & Min & 25th & 50th & 75th & Max & \\
\hline Fries & 49 & $1(3)$ & 0 & 0 & 0 & 1 & 8 & $0(0)$ & 0 & 0 & 0 & 0 & 1 & $14 \%$ \\
\hline Hash Browns and Patties & 19 & $0(1)$ & 0 & 0 & 0 & 1 & 2 & $0(0)$ & 0 & 0 & 0 & 0 & 1 & $42 \%$ \\
\hline Mashed and Scalloped & 58 & $2(1)$ & 0 & 1 & 1 & 2 & 9 & $1(1)$ & 0 & 0 & 0 & 0 & 8 & $19 \%$ \\
\hline Prepared Salads & 61 & $5(4)$ & 0 & 2 & 3 & 6 & 16 & $4(4)$ & 0 & 1 & 3 & 6 & 15 & $75 \%$ \\
\hline Coleslaw & 6 & $14(1)$ & 12 & 13 & 14 & 15 & 16 & $13(1)$ & 12 & 12 & 13 & 15 & 15 & $95 \%$ \\
\hline Fish and Meat Salad & 11 & $3(3)$ & 0 & 2 & 2 & 3 & 9 & $3(3)$ & 0 & 2 & 2 & 3 & 9 & $100 \%$ \\
\hline Grain-Based Salad & 6 & $4(6)$ & 1 & 1 & 2 & 6 & 15 & $3(6)$ & 0 & 0 & 1 & 5 & 14 & $40 \%$ \\
\hline Pasta Salad & 8 & $4(2)$ & 1 & 2 & 5 & 6 & 6 & $3(2)$ & 0 & 1 & 3 & 5 & 5 & $58 \%$ \\
\hline Potato Salad & 8 & $4(1)$ & 3 & 3 & 4 & 5 & 6 & $4(1)$ & 2 & 3 & 3 & 4 & 6 & $82 \%$ \\
\hline Vegetable Salad & 22 & $5(4)$ & 0 & 2 & 3 & 9 & 12 & $4(4)$ & 0 & 1 & 2 & 8 & 11 & $72 \%$ \\
\hline Refrigerated or Frozen & 775 & $3(2)$ & 0 & 1 & 2 & 4 & 20 & $2(2)$ & 0 & 0 & 1 & 2 & 19 & $46 \%$ \\
\hline $170-285 \mathrm{~g}$ & 290 & $2(2)$ & 0 & 1 & 2 & 3 & 12 & $1(2)$ & 0 & 0 & 1 & 2 & 12 & $43 \%$ \\
\hline Less than $170 \mathrm{~g}$ & 381 & $3(3)$ & 0 & 1 & 2 & 4 & 20 & $2(2)$ & 0 & 0 & 1 & 2 & 19 & $45 \%$ \\
\hline More than $285 \mathrm{~g}$ & 104 & $3(2)$ & 0 & 1 & 2 & 4 & 16 & $2(3)$ & 0 & 0 & 2 & 3 & 15 & $56 \%$ \\
\hline Shelf-Stable, Grain-Based Dishes & 310 & $2(2)$ & 0 & 1 & 2 & 3 & 9 & $1(1)$ & 0 & 0 & 0 & 1 & 8 & $36 \%$ \\
\hline Pasta and Noodles & 177 & $3(1)$ & 0 & 2 & 3 & 4 & 7 & $1(1)$ & 0 & 0 & 1 & 1 & 5 & $25 \%$ \\
\hline Rice and Grains & 116 & $1(1)$ & 0 & 0 & 1 & 1 & 9 & $1(1)$ & 0 & 0 & 0 & 1 & 8 & $51 \%$ \\
\hline Stuffing & 17 & $2(1)$ & 0 & 2 & 2 & 3 & 5 & $2(1)$ & 0 & 1 & 2 & 2 & 4 & $79 \%$ \\
\hline Nuts and Seeds & 205 & $5(4)$ & 0 & 3 & 4 & 7 & 28 & $1(4)$ & 0 & 0 & 0 & 0 & 25 & $11 \%$ \\
\hline Butters, Pastes and Creams & 78 & $8(6)$ & 0 & 6 & 7 & 8 & 28 & $3(5)$ & 0 & 0 & 0 & 3 & 25 & $30 \%$ \\
\hline Other than Peanut Butter & 28 & $4(4)$ & 0 & 0 & 3 & 7 & 13 & $2(4)$ & 0 & 0 & 0 & 2 & 10 & $28 \%$ \\
\hline Peanut Butter & 50 & $9(5)$ & 6 & 7 & 7 & 13 & 28 & $4(6)$ & 0 & 0 & 2 & 9 & 25 & $31 \%$ \\
\hline Nuts and Seeds & 127 & $4(2)$ & 0 & 2 & 3 & 4 & 13 & $0(0)$ & 0 & 0 & 0 & 0 & 0 & $0 \%$ \\
\hline Nut and Seed Flours & 7 & $7(5)$ & 0 & 4 & 7 & 13 & 13 & $0(0)$ & 0 & 0 & 0 & 0 & 0 & $0 \%$ \\
\hline Nuts and Seeds, Not for Snacking & 120 & $3(2)$ & 0 & 2 & 3 & 4 & 8 & $0(0)$ & 0 & 0 & 0 & 0 & 0 & $0 \%$ \\
\hline Other Foods and Beverages & 274 & $6(13)$ & 0 & 0 & 0 & 8 & 100 & $6(13)$ & 0 & 0 & 0 & 6 & 100 & $87 \%$ \\
\hline Baking Misc. (e.g., yeast, baking soda) & 15 & $0(0)$ & 0 & 0 & 0 & 0 & 0 & $0(0)$ & 0 & 0 & 0 & 0 & 0 & \\
\hline Seasoning, Topping, Breading Mix & 259 & $8(14)$ & 0 & 0 & 0 & 13 & 100 & $7(14)$ & 0 & 0 & 0 & 9 & 100 & $86 \%$ \\
\hline Sauces, Dips and Condiments & 1204 & $11(14)$ & 0 & 2 & 5 & 17 & 70 & $10(15)$ & 0 & 0 & 2 & 17 & 70 & $63 \%$ \\
\hline Condiments & 291 & $18(17)$ & 0 & 0 & 19 & 33 & 66 & $18(17)$ & 0 & 0 & 17 & 31 & 66 & $96 \%$ \\
\hline Barbecue and Steak Sauce & 115 & $30(14)$ & 0 & 21 & 31 & 38 & 66 & $30(14)$ & 0 & 21 & 31 & 38 & 66 & $100 \%$ \\
\hline Ketchup & 24 & $24(8)$ & 7 & 20 & 27 & 27 & 33 & $18(8)$ & 0 & 14 & 21 & 21 & 29 & $70 \%$ \\
\hline Mustard & 54 & $9(15)$ & 0 & 0 & 0 & 20 & 60 & $9(15)$ & 0 & 0 & 0 & 20 & 60 & $100 \%$ \\
\hline Other Condiments (e.g., hot sauce) & 98 & $9(12)$ & 0 & 0 & 3 & 17 & 60 & $9(12)$ & 0 & 0 & 3 & 17 & 60 & $97 \%$ \\
\hline Dips & 259 & $3(3)$ & 0 & 0 & 3 & 4 & 25 & $1(3)$ & 0 & 0 & 0 & 1 & 23 & $24 \%$ \\
\hline Dips and Salsa & 210 & $4(3)$ & 0 & 3 & 3 & 5 & 25 & $1(3)$ & 0 & 0 & 0 & 1 & 23 & $23 \%$ \\
\hline Hummus and Legume Dips & 49 & $1(2)$ & 0 & 0 & 0 & 3 & 10 & $1(2)$ & 0 & 0 & 0 & 0 & 9 & $29 \%$ \\
\hline
\end{tabular}


Table 3. Cont.

\begin{tabular}{|c|c|c|c|c|c|c|c|c|c|c|c|c|c|c|}
\hline \multirow{2}{*}{$\begin{array}{l}\text { Food Group, Subcategory, and } \\
\text { Minor Category }\end{array}$} & \multirow{2}{*}{$n$} & \multicolumn{6}{|c|}{ Total Sugar (g/100 g or $100 \mathrm{~mL}$ ) } & \multicolumn{6}{|c|}{ Free Sugar (g/100 g or $100 \mathrm{~mL}$ ) } & \multirow{2}{*}{$\begin{array}{l}\text { Free Sugar as a } \\
\text { Percent of Total } \\
\text { Sugar }^{2}\end{array}$} \\
\hline & & $\bar{X}(\mathrm{SD})$ & Min & 25th & 50th & 75th & Max & $\bar{X}(\mathrm{SD})$ & Min & 25th & 50th & 75th & Max & \\
\hline Sauces & 654 & $11(14)$ & 0 & 2 & 5 & 13 & 70 & $10(15)$ & 0 & 0 & 3 & 13 & 70 & $65 \%$ \\
\hline Curry Paste & 27 & $6(4)$ & 0 & 3 & 5 & 7 & 16 & $3(4)$ & 0 & 0 & 0 & 5 & 14 & $37 \%$ \\
\hline Gravy and Cooking Sauce & 188 & $8(12)$ & 0 & 0 & 3 & 9 & 48 & $8(12)$ & 0 & 0 & 3 & 8 & 48 & $79 \%$ \\
\hline Marinades & 60 & $15(14)$ & 0 & 5 & 11 & 22 & 50 & $15(14)$ & 0 & 5 & 11 & 22 & 50 & $100 \%$ \\
\hline Pesto & 15 & $3(6)$ & 0 & 0 & 0 & 5 & 20 & $0(1)$ & 0 & 0 & 0 & 0 & 2 & $29 \%$ \\
\hline Soya and Oriental Sauce & 61 & $16(17)$ & 0 & 7 & 12 & 24 & 70 & $16(17)$ & 0 & 7 & 12 & 24 & 70 & $98 \%$ \\
\hline Sweet Sauce (e.g., plum sauce) & 73 & $35(14)$ & 0 & 27 & 34 & 43 & 63 & $35(14)$ & 0 & 27 & 34 & 43 & 63 & $100 \%$ \\
\hline Tomato Sauce & 198 & $4(2)$ & 0 & 3 & 5 & 5 & 8 & $2(2)$ & 0 & 0 & 2 & 3 & 6 & $37 \%$ \\
\hline White Sauce & 32 & $3(2)$ & 2 & 2 & 3 & 4 & 7 & $1(2)$ & 0 & 0 & 1 & 1 & 5 & $26 \%$ \\
\hline Snacks & 854 & $10(16)$ & 0 & 2 & 4 & 8 & 83 & $5(10)$ & 0 & 0 & 0 & 4 & 65 & $44 \%$ \\
\hline Chips, Corn and Rice Snacks & 412 & $4(5)$ & 0 & 0 & 3 & 5 & 35 & $3(5)$ & 0 & 0 & 1 & 3 & 34 & $59 \%$ \\
\hline Extruded Snacks (e.g., cheese puffs) & 90 & $6(8)$ & 0 & 2 & 5 & 7 & 35 & $5(8)$ & 0 & 0 & 1 & 6 & 34 & $52 \%$ \\
\hline Flavoured Chips & 194 & $4(3)$ & 0 & 2 & 4 & 4 & 15 & $3(3)$ & 0 & 1 & 3 & 3 & 14 & $68 \%$ \\
\hline Plain Chips & 116 & $2(4)$ & 0 & 0 & 0 & 2 & 30 & $1(2)$ & 0 & 0 & 0 & 0 & 17 & $32 \%$ \\
\hline Savoury Snack Mixes & 12 & $5(2)$ & 2 & 3 & 5 & 6 & 9 & $3(2)$ & 1 & 2 & 4 & 5 & 8 & $65 \%$ \\
\hline Ethnic Snacks & 21 & $7(12)$ & 0 & 2 & 4 & 8 & 54 & $5(12)$ & 0 & 0 & 0 & 6 & 53 & $38 \%$ \\
\hline Fruit Snacks (e.g., apple chips, fruit leather) & 40 & $61(24)$ & 6 & 49 & 72 & 78 & 83 & $9(14)$ & 0 & 0 & 0 & 24 & 37 & $12 \%$ \\
\hline Meat Snacks & 42 & $9(11)$ & 0 & 0 & 4 & 18 & 43 & $9(11)$ & 0 & 0 & 4 & 18 & 43 & $96 \%$ \\
\hline Meat and Poultry Jerky & 20 & $19(9)$ & 7 & 12 & 18 & 21 & 43 & $19(9)$ & 7 & 12 & 18 & 21 & 43 & $100 \%$ \\
\hline Meat and Poultry Sticks & 22 & $1(1)$ & 0 & 0 & 0 & 2 & 4 & $1(1)$ & 0 & 0 & 0 & 2 & 4 & $88 \%$ \\
\hline Nuts and Seeds & 225 & $13(13)$ & 0 & 4 & 6 & 22 & 52 & $4(9)$ & 0 & 0 & 0 & 3 & 41 & $15 \%$ \\
\hline Mix w/ Fruit, Chocolate, Candy & 80 & $28(10)$ & 10 & 20 & 28 & 33 & 52 & $12(12)$ & 0 & 0 & 11 & 17 & 41 & $34 \%$ \\
\hline Mix w/o Fruit, Chocolate, Candy & 145 & $5(2)$ & 0 & 4 & 4 & 6 & 14 & $0(1)$ & 0 & 0 & 0 & 0 & 8 & $5 \%$ \\
\hline Popcorn & 80 & $9(17)$ & 0 & 0 & 0 & 6 & 65 & $9(17)$ & 0 & 0 & 0 & 4 & 65 & $63 \%$ \\
\hline Plain/Savoury & 61 & $1(2)$ & 0 & 0 & 0 & 2 & 6 & $0(1)$ & 0 & 0 & 0 & 0 & 6 & $30 \%$ \\
\hline Sweet & 19 & $35(17)$ & 0 & 24 & 34 & 42 & 65 & $35(17)$ & 0 & 24 & 34 & 42 & 65 & $100 \%$ \\
\hline Pretzels & 34 & $12(15)$ & 0 & 2 & 4 & 14 & 43 & $11(15)$ & 0 & 1 & 3 & 13 & 42 & $80 \%$ \\
\hline Coated or Filled & 14 & $26(14)$ & 8 & 14 & 23 & 43 & 43 & $25(14)$ & 7 & 13 & 22 & 42 & 42 & $97 \%$ \\
\hline Plain & 20 & $2(2)$ & 0 & 2 & 2 & 4 & 5 & $2(1)$ & 0 & 0 & 1 & 3 & 4 & $66 \%$ \\
\hline Soups & 464 & $1(1)$ & 0 & 0 & 1 & 2 & 7 & $1(1)$ & 0 & 0 & 0 & 1 & 7 & $52 \%$ \\
\hline Bouillon and Broth & 110 & $0(0)$ & 0 & 0 & 0 & 0 & 1 & $0(0)$ & 0 & 0 & 0 & 0 & 1 & $94 \%$ \\
\hline Broth & 56 & $0(0)$ & 0 & 0 & 0 & 0 & 1 & $0(0)$ & 0 & 0 & 0 & 0 & 1 & $90 \%$ \\
\hline Dry Mix & 39 & $0(0)$ & 0 & 0 & 0 & 0 & 1 & $0(0)$ & 0 & 0 & 0 & 0 & 1 & $100 \%$ \\
\hline Liquid Concentrates & 15 & $0(0)$ & 0 & 0 & 0 & 0 & 0 & $0(0)$ & 0 & 0 & 0 & 0 & 0 & $100 \%$ \\
\hline Canned Condensed Soup & 76 & $1(1)$ & 0 & 0 & 1 & 2 & 6 & $1(1)$ & 0 & 0 & 0 & 1 & 6 & $45 \%$ \\
\hline Cream or Cheese & 32 & $1(1)$ & 0 & 0 & 1 & 2 & 2 & $0(0)$ & 0 & 0 & 0 & 0 & 1 & $12 \%$ \\
\hline Non-Cream & 44 & $2(2)$ & 0 & 0 & 1 & 2 & 6 & $1(2)$ & 0 & 0 & 1 & 2 & 6 & $69 \%$ \\
\hline Dry Soup Mix & 55 & $1(1)$ & 0 & 0 & 1 & 1 & 4 & $0(1)$ & 0 & 0 & 0 & 1 & 3 & $37 \%$ \\
\hline Cream or Cheese & 15 & $2(1)$ & 0 & 1 & 2 & 3 & 3 & $1(1)$ & 0 & 1 & 1 & 1 & 2 & $53 \%$ \\
\hline Non-Cream & 40 & $1(1)$ & 0 & 0 & 0 & 1 & 4 & $0(1)$ & 0 & 0 & 0 & 0 & 3 & $30 \%$ \\
\hline
\end{tabular}


Table 3. Cont

\begin{tabular}{|c|c|c|c|c|c|c|c|c|c|c|c|c|c|c|}
\hline \multirow{2}{*}{$\begin{array}{l}\text { Food Group, Subcategory, and } \\
\text { Minor Category }\end{array}$} & \multirow{2}{*}{$n$} & \multicolumn{6}{|c|}{ Total Sugar (g/100 g or $100 \mathrm{~mL})$} & \multicolumn{6}{|c|}{ Free Sugar (g/100 g or $100 \mathrm{~mL})$} & \multirow{2}{*}{$\begin{array}{l}\text { Free Sugar as a } \\
\text { Percent of Total } \\
\text { Sugar }^{2}\end{array}$} \\
\hline & & $\bar{X}(\mathrm{SD})$ & Min & 25th & 50th & 75th & Max & $\bar{X}(\mathrm{SD})$ & Min & 25th & 50th & 75th & Max & \\
\hline Fresh and Instant Oriental Noodle & 76 & $1(1)$ & 0 & 0 & 1 & 1 & 3 & $1(1)$ & 0 & 0 & 1 & 1 & 3 & $87 \%$ \\
\hline Ready-to-Serve Soup & 147 & $2(1)$ & 0 & 1 & 2 & 3 & 7 & $1(1)$ & 0 & 0 & 0 & 2 & 7 & $30 \%$ \\
\hline Cream or Cheese & 28 & $2(2)$ & 0 & 0 & 1 & 4 & 7 & $2(2)$ & 0 & 0 & 1 & 3 & 7 & $65 \%$ \\
\hline Non-Cream Soup & 119 & $2(1)$ & 0 & 1 & 2 & 3 & 6 & $1(1)$ & 0 & 0 & 0 & 1 & 5 & $23 \%$ \\
\hline Sugars and Sweets & 776 & $51(17)$ & 0 & 43 & 50 & 60 & 100 & $51(17)$ & 0 & 43 & 50 & 60 & 100 & $100 \%$ \\
\hline Confectionery & 469 & $51(16)$ & 0 & 45 & 51 & 60 & 100 & $51(16)$ & 0 & 45 & 51 & 60 & 100 & $100 \%$ \\
\hline $\begin{array}{l}\text { Baking Candies (e.g., sprinkles, } \\
\text { chocolate chips) }\end{array}$ & 33 & $53(19)$ & 0 & 47 & 53 & 62 & 100 & $53(19)$ & 0 & 47 & 53 & 62 & 100 & $100 \%$ \\
\hline Breath Mints & 6 & $94(5)$ & 88 & 93 & 93 & 100 & 100 & $94(5)$ & 88 & 93 & 93 & 100 & 100 & $100 \%$ \\
\hline $\begin{array}{l}\text { Candies (e.g., licorice, gummies, } \\
\text { jelly beans) }\end{array}$ & 151 & $56(12)$ & 6 & 48 & 55 & 63 & 100 & $56(12)$ & 6 & 48 & 55 & 63 & 100 & $100 \%$ \\
\hline Chocolate and Candy Bars & 257 & $46(15)$ & 0 & 42 & 49 & 54 & 80 & $46(15)$ & 0 & 42 & 49 & 54 & 80 & $100 \%$ \\
\hline Hard Candies & 9 & $70(17)$ & 48 & 61 & 68 & 80 & 94 & $70(17)$ & 48 & 61 & 68 & 80 & 94 & $100 \%$ \\
\hline Marshmallows & 13 & $56(4)$ & 52 & 52 & 54 & 57 & 67 & $56(4)$ & 52 & 52 & 54 & 57 & 67 & $100 \%$ \\
\hline Sugar & 7 & $98(4)$ & 89 & 100 & 100 & 100 & 100 & $98(4)$ & 89 & 100 & 100 & 100 & 100 & $100 \%$ \\
\hline Icing Sugar & 1 & $89(0)$ & 89 & 89 & 89 & 89 & 89 & $89(0)$ & 89 & 89 & 89 & 89 & 89 & $100 \%$ \\
\hline Sugar & 6 & $100(0)$ & 100 & 100 & 100 & 100 & 100 & $100(0)$ & 100 & 100 & 100 & 100 & 100 & $100 \%$ \\
\hline Sweet Condiments & 300 & $50(17)$ & 0 & 40 & 50 & 60 & 81 & $50(17)$ & 0 & 40 & 50 & 60 & 81 & $100 \%$ \\
\hline Bread Spreads (e.g., chocolate spread) & 13 & $52(15)$ & 25 & 47 & 50 & 58 & 79 & $52(15)$ & 25 & 47 & 50 & 58 & 79 & $100 \%$ \\
\hline Fruit Preserve Spreads (e.g., jam, jelly) & 187 & $45(14)$ & 0 & 35 & 45 & 55 & 75 & $45(14)$ & 0 & 35 & 45 & 55 & 75 & $100 \%$ \\
\hline Honey and Molasses & 38 & $76(9)$ & 45 & 76 & 80 & 80 & 80 & $76(9)$ & 45 & 76 & 80 & 80 & 80 & $100 \%$ \\
\hline Syrups & 62 & $49(16)$ & 3 & 40 & 50 & 61 & 81 & $49(16)$ & 3 & 40 & 50 & 61 & 81 & $100 \%$ \\
\hline Vegetables & 957 & $3(6)$ & 0 & 0 & 2 & 4 & 40 & $2(5)$ & 0 & 0 & 0 & 0 & 40 & $16 \%$ \\
\hline Canned Vegetables and Legumes & 460 & $2(2)$ & 0 & 0 & 1 & 3 & 29 & $0(1)$ & 0 & 0 & 0 & 0 & 6 & $15 \%$ \\
\hline Canned Tomatoes & 93 & $3(1)$ & 1 & 2 & 3 & 4 & 6 & $0(1)$ & 0 & 0 & 0 & 0 & 3 & $9 \%$ \\
\hline Other Canned Vegetables & 367 & $2(2)$ & 0 & 0 & 1 & 2 & 29 & $0(1)$ & 0 & 0 & 0 & 0 & 6 & $17 \%$ \\
\hline Dried Legumes & 86 & $3(2)$ & 0 & 1 & 2 & 3 & 11 & $0(0)$ & 0 & 0 & 0 & 0 & 0 & $0 \%$ \\
\hline Fresh Vegetables & 54 & $3(6)$ & 0 & 0 & 2 & 3 & 40 & $0(2)$ & 0 & 0 & 0 & 0 & 18 & $3 \%$ \\
\hline Frozen Vegetables & 155 & $3(2)$ & 0 & 2 & 2 & 4 & 14 & $0(0)$ & 0 & 0 & 0 & 0 & 4 & $2 \%$ \\
\hline Frozen Vegetables w/ Sauce & 9 & $2(2)$ & 0 & 2 & 2 & 3 & 5 & $1(1)$ & 0 & 0 & 1 & 1 & 4 & $40 \%$ \\
\hline Frozen Vegetables w/o Sauce & 146 & $3(2)$ & 0 & 2 & 2 & 4 & 14 & $0(0)$ & 0 & 0 & 0 & 0 & 0 & $0 \%$ \\
\hline Vegetable Paste & 20 & $6(5)$ & 0 & 0 & 9 & 9 & 13 & $0(0)$ & 0 & 0 & 0 & 0 & 0 & $0 \%$ \\
\hline Tomato Paste & 12 & $10(1)$ & 9 & 9 & 9 & 9 & 13 & $0(0)$ & 0 & 0 & 0 & 0 & 0 & $0 \%$ \\
\hline Vegetable and Herb Paste & 8 & $0(0)$ & 0 & 0 & 0 & 0 & 0 & $0(0)$ & 0 & 0 & 0 & 0 & 0 & \\
\hline Pickled Vegetables & 182 & $8(10)$ & 0 & 0 & 3 & 13 & 40 & $7(10)$ & 0 & 0 & 0 & 13 & 40 & $57 \%$ \\
\hline Sour or Spicy & 126 & $2(4)$ & 0 & 0 & 0 & 3 & 29 & $0(1)$ & 0 & 0 & 0 & 0 & 9 & $11 \%$ \\
\hline Sweet & 56 & $21(7)$ & 0 & 14 & 20 & 27 & 40 & $21(7)$ & 0 & 14 & 20 & 27 & 40 & $100 \%$ \\
\hline TOTAL & 15259 & $11(16)$ & 0 & 1 & 4 & 13 & 160 & $9(16)$ & 0 & 0 & 1 & 11 & 160 & $62 \%$ \\
\hline
\end{tabular}

${ }^{1}$ All values presented represent products in their "as consumed" form, prepared according to package directions; ${ }^{2}$ Free sugar as a percent of total sugar was calculated for each product $(n=15,259)$ and the average of those results is presented here; ${ }^{3}$ Total and free sugar contents for beverages and desserts presented as $g$ per $100 \mathrm{~mL} ;{ }^{4}$ Maximum total sugar content exceeds $100 \mathrm{~g}$ per $100 \mathrm{~mL}$ due to rounding of total sugar declaration on products with small serving sizes in desserts food group. Abbreviations: NFt = Nutrition Facts table; $\bar{X}=$ mean; $\mathrm{SD}=$ standard deviation; $\mathrm{w} / \mathrm{=}$ with; $\mathrm{w} / \mathrm{o}=$ without 


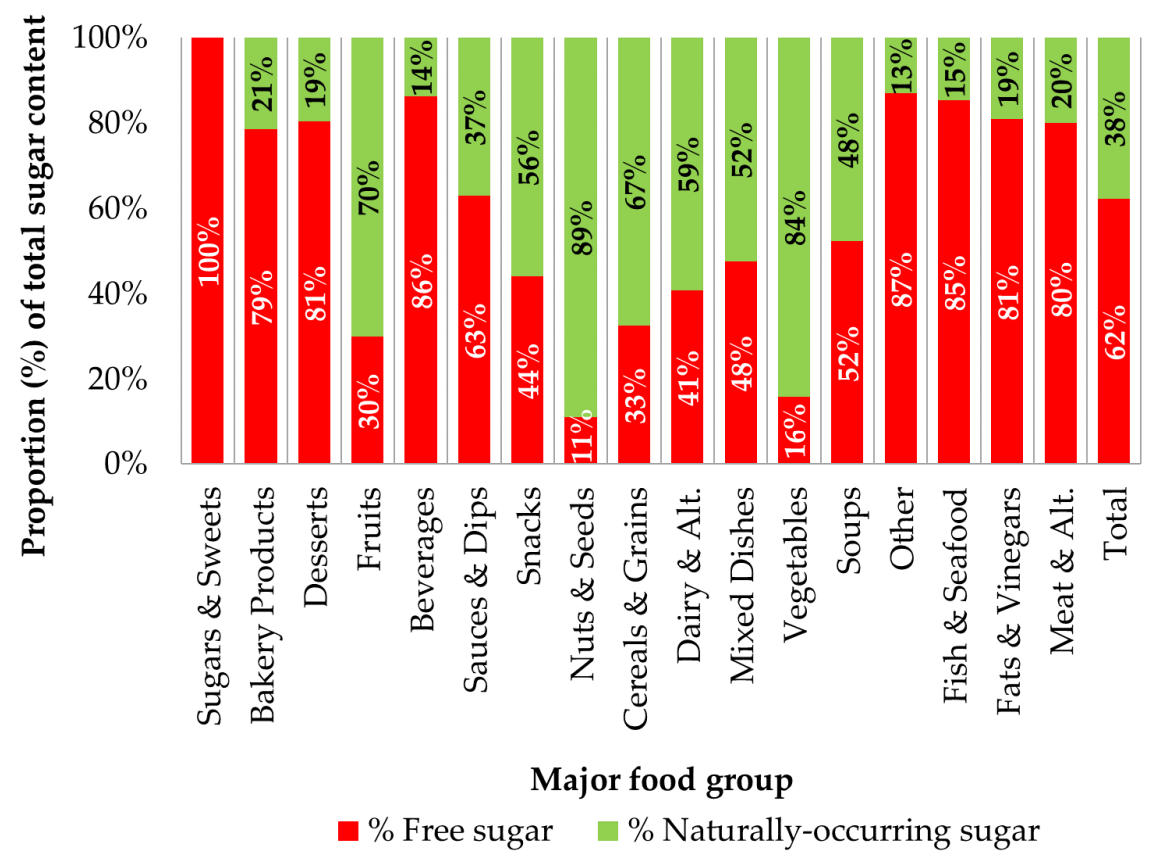

Figure 3. Free sugar and naturally-occurring sugar as a proportion (\%) of total sugar by major food group and overall $(n=15,259)$. Free sugar and naturally-occurring sugar as a percent of total sugar was calculated for each product and the average of those results is presented here.

\subsection{Contribution of Free Sugar to Total Calories}

Free sugar contributed on average $20 \%$ of calories in the prepackaged foods and beverages evaluated (Figure 4 ), with content $\geq 10 \%$ of calories in seven of the 17 major food groups, including beverages $(70 \%)$, sugars and sweets $(62 \%)$, and desserts $(41 \%)$.

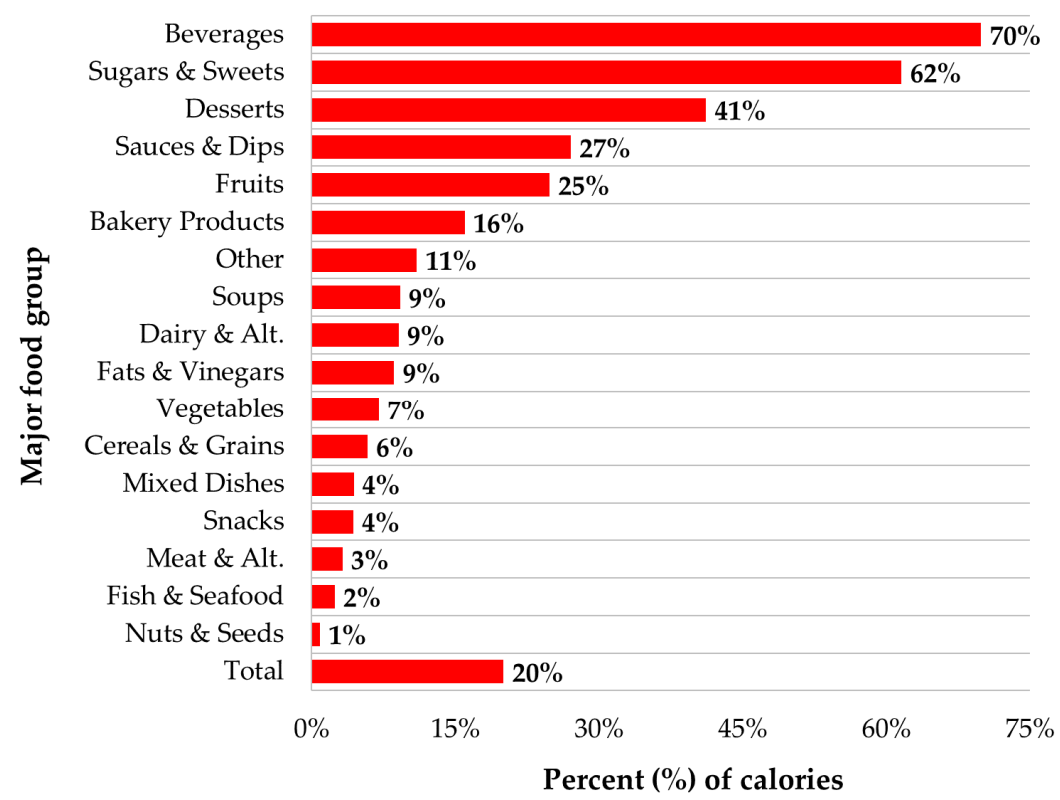

Figure 4. Free sugar as a percent of calories, by major food group and overall $(n=15,259)$. 


\section{Discussion}

With a number of recommendations to enact policies and initiate programs that support limiting sugar intakes, it is imperative that baseline information on the types and amounts of sugar in Canadian foods and beverages be available for researchers, policy-makers, healthcare practitioners and consumers to make evidence-based decisions. This study was conducted to meet this need and is the first to systematically calculate free sugar content and to report on the total and free sugar contents and the use of FSI and sweeteners in a large representative sample of Canadian prepackaged foods and beverages.

Alarmingly, free sugar in products contributed an average of $20 \%$ of calories in prepackaged foods and beverages, which is in excess of WHO free su34gar and US Dietary Guidelines added sugar intake recommendations at a maximum of $10 \%$ of calories $[9,10]$. Consumption of products with excessive free sugar contents, enhances the likelihood of exceeding these recommendations [38]. Some of the more sugar-dense food groups identified in this study, foods such as sweet bakery products, frozen desserts, confectionery, and sugar-sweetened beverages, are not recommended in Eating Well with Canada's Food Guide [39]; yet these "other foods" contributed more than one-third (34.7\%) of the total sugar Canadians consumed in 2004 [24,39].

This study identified 152 different names for FSI used in Canadian Ingredient Lists, highlighting the challenge faced by consumers trying to limit their intakes of free sugar. These FSI were ubiquitously found throughout the food supply and were present in every major food group. Data on FSI use in Canadian foods $(65.4 \%)$, are slightly lower than that reported in the US where $74 \%$ of packaged foods were reported to contain added sugar ingredients in 2005-2009 [40].

Results of this study also identified that sweeteners were used in less than $5 \%$ of products. Not surprisingly, they are most often used in food groups with the highest total and free sugar contents. Efforts to reduce added or free sugar intakes have raised concerns that reformulation will not result in a reduction in calories (e.g., due to an increase in refined starches, fats) [41], or will increase the use of artificial sweeteners [42,43]. The evidence of the health effects or benefits of sweetener use in the long-term is inconclusive [42,43]. Thus, some recommendations to reduce sugar content of prepackaged foods stipulate that this should not be met with the subsequent addition of sweeteners $[8,22]$.

The data presented here can be useful to support several interventions aimed at reducing intakes of free sugar. Firstly, reformulation of existing products and the development of new products to be lower in sugar have been suggested as ways to decrease the health burden associated with excess free sugar consumption [44]. This strategy, similar to the sodium reduction strategies in Canada [31] and other countries could likely be repeated for free sugar [22]. The data provided here would support such a strategy and shows that there is a wide range of free sugar content within a food category, demonstrating that products with lower free sugar contents are achievable, feasible, palatable, and sellable as shown in Table 3. This type of intra-category assessment of distributions can be used to develop the benchmarks needed for concerted efforts at free sugar reduction. Secondly, data on the free sugar content of prepackaged foods can be linked to national dietary intake surveys to provide the first evaluation of Canadian free sugar consumption. Subsequently, consumption data can be used to predict and monitor health outcomes associated with varying levels of free sugar intakes. Thirdly, the data on sweetener use and FSI use provided here can act as a baseline by which to compare future trends. Additionally, this data can be used to support consumer educational efforts to emphasize the many names for FSI, thereby helping consumers to more easily identify products that contain free sugar as well as the main food sources. One novel feature of the recently proposed Canadian nutrition labelling changes to address this concern is the proposal to group all sugar based ingredients in brackets after the word "Sugars" and be placed in the ingredient list in descending order according to the combined weight rather than scattered throughout the Ingredient List [45].

Limitations of this study include the use of declared sugar contents from the NFt, rather than laboratory analyses. NFt declarations are subject to the Canadian Food Inspection Agency's rounding rules and can vary up to $20 \%$ from the actual analyzed value [46]. Given the vastness of the database, 
analysis of each product was not a feasible option. However, a study evaluating the accuracy of the declared nutrient contents of 1000 Canadian foods found only $13 \%$ of foods with unsatisfactory values (>20\% difference from analyzed) for sugar contents [47]. Additionally, there are no chemical analyses available to differentiate free or added sugar from total sugar content. To account for this, the calculation of free sugar contents was based on a similar algorithm developed by Louie and colleagues [36], to estimate added sugar contents in the Australian food supply, that has been shown to have high levels of inter-researcher repeatability [36]. The most subjective step in the U of T free sugar algorithm, Step 5, where substitute added sugar values are chosen, was done by two people and consensus was reached for any discrepancies; however, this step was only required for $2.6 \%$ of foods. Finally, the FLIP 2013 database did not include all prepackaged foods and beverages available in Canada, but rather a systematically collected and large representative subset, comprising over three-quarters of the Canadian grocery retail market share.

\section{Conclusions}

In summary, this is the first study in Canada to calculate free sugar contents and these data provide the first detailed overview of FSI and sweetener use, and of total and free sugar contents of Canadian prepackaged foods and beverages. Using the detailed free sugar algorithm and the information from the NFt and Ingredient List, free sugar content was calculated for 96.5\% of the foods and only imputed for 3.5\%. The method described here can be employed for use on other large branded food databases. Findings can be used to inform, monitor, and evaluate interventions to limit excess sugar consumption, and indicate areas of concern for reformulation or educational efforts. The extensive data provided in this study can be incorporated into food composition databases and can be used to measure free sugar intakes with national nutrition surveys where it is currently not available [24], and determine intakes, particularly for vulnerable groups such as children and adolescents [8,25], compared to recommendations from the WHO [9].

Acknowledgments: This research was supported by the Canadian Institute for Health Research (CIHR) Strategic Operating Grant (201103SOK-118150) and the Canadian Stroke Network (201103SOK-01194-000) and One Sweet Film (M.R.L). J.T.B. is supported by the CIHR Strategic Training Grant in Population Intervention for Chronic Disease Prevention (TGF-53893) and the CIHR Collaborative Training Program in Public Health Policy. M.R.L. is the Earle W. McHenry professor and is supported by the chair endowed unrestricted research funds, University of Toronto. The authors have no conflicts of interest to declare. The funding sponsors had no role in the design of the study; in the collection, analyses, or interpretation of data; in the writing of the manuscript, and in the decision to publish the results. The authors would also like to acknowledge the support of Katherine Jefferson, who helped to categorize products, verify nutrient contents, and conduct weight/volume conversions. Lauren Renlund, a MPH student at the University of Toronto who assisted with free sugar calculations. Developers and staff at Plank Design and Nathalie Bibeau from One Sweet Film Inc. who enabled and created a program that automatically identified free sugar ingredients in FLIP 2013.

Author Contributions: J.T.B. and M.R.L. conceived and designed the overall research plan. A.S. coordinated data collection. C.M.M. compiled data on free sugar ingredient use. J.T.B. conducted research, analyzed data and wrote the manuscript. All authors were responsible for final content.

Conflicts of Interest: The authors declare no conflict of interest.

\section{References}

1. Yang, Q.; Zhang, Z.; Gregg, E.W.; Flanders, W.; Merritt, R.; Hu, F.B. Added sugar intake and cardiovascular diseases mortality among us adults. JAMA Intern. Med. 2014, 174, 516-524. [CrossRef] [PubMed]

2. Sheiham, A.; James, W.P. A new understanding of the relationship between sugars, dental caries and fluoride use: Implications for limits on sugars consumption. Public Health Nutr. 2014, 17, 2176-2184. [CrossRef] [PubMed]

3. Malik, V.S.; Popkin, B.M.; Bray, G.A.; Despres, J.P.; Willett, W.C.; Hu, F.B. Sugar-sweetened beverages and risk of metabolic syndrome and type 2 diabetes: A meta-analysis. Diabetes Care 2010, 33, 2477-2483. [CrossRef] [PubMed] 
4. Te Morenga, L.A.; Howatson, A.J.; Jones, R.M.; Mann, J. Dietary sugars and cardiometabolic risk: Systematic review and meta-analyses of randomized controlled trials of the effects on blood pressure and lipids. Am. J. Clin. Nutr. 2014, 100, 65-79. [CrossRef] [PubMed]

5. Te Morenga, L.; Mallard, S.; Mann, J. Dietary sugars and body weight: Systematic review and meta-analyses of randomised controlled trials and cohort studies. BMJ 2013, 346, e7492. [CrossRef] [PubMed]

6. Li, Y.; Hruby, A.; Bernstein, A.M.; Ley, S.H.; Wang, D.D.; Chiuve, S.E.; Sampson, L.; Rexrode, K.M.; Rimm, E.B.; Willett, W.C.; et al. Saturated fats compared with unsaturated fats and sources of carbohydrates in relation to risk of coronary heart disease: A prospective cohort study. J. Am. Coll. Cardiol. 2015, 66, 1538-1548. [CrossRef] [PubMed]

7. Canadian Diabetes Association. Position on Sugars, 2015. Available online: http://www.diabetes.ca/aboutcda/public-policy-position-statements/sugars (accessed on 25 January 2016).

8. Heart and Stroke Foundation Canada. Position Statement-Sugar, Heart Disease and Stroke, 2014. Available online: http:/ /www.heartandstroke.com/atf/cf/\%7B99452D8B-E7F1-4BD6-A57D-B136CE6C95BF\%7D/ Sugar-Eng.pdf (accessed on 3 October 2014).

9. World Health Organization. Guideline: Sugars Intake for Adults and Children, 2015. Available online: http:/ /apps.who.int/iris/bitstream/10665/149782/1/9789241549028_eng.pdf?ua=1 (accessed on 10 December 2015).

10. United States Department of Health and Human Services and United States Department of Agriculture. 2015-2020 Dietary Guidelines for Americans, 8th ed.; 2015. Available online: http://health.gov/ dietaryguidelines/2015/guidelines/ (accessed on 12 January 2016).

11. Public Health England. Sugar Reduction: From Evidence to Action, $2015 . \quad$ Available online: https://www.gov.uk/government/uploads/system/uploads/attachment_data/file/470179/ Sugar_reduction_The_evidence_for_action.pdf (accessed on 25 October 2015).

12. United Nations General Assembly. Prevention and Control of Non-Communicable Diseases, 2011. Available online: http://www.un.org/en/ga/search/view_doc.asp?symbol=A/66/83\&Lang=E (accessed on 18 August 2014).

13. Institute of Medicine. Dietary Reference Intakes for Energy, Carbohydrate, Fiber, Fat, Fatty Acids, Cholesterol, Protein and Amino Acids; National Academy Press: Washington, DC, USA, 2001.

14. Sigman-Grant, M.; Morita, J. Defining and interpreting intakes of sugars. Am. J. Clin. Nutr. 2003, 78, 815-826.

15. Neal, B.; Sacks, G.; Swinburn, B.; Vandevijvere, S.; Dunford, E.; Snowdon, W.; Webster, J.; Barquera, S.; Friel, S.; Hawkes, C. Monitoring the levels of important nutrients in the food supply. Obes. Rev. 2013, 14, 49-58. [CrossRef] [PubMed]

16. Food and Drug Administration. Food Labeling: Revision of the Nutrition and Supplement Facts Labels, 2016. Available online: https:/ / www.federalregister.gov/documents/2014/03/03/2014-04387/food-labelingrevision-of-the-nutrition-and-supplement-facts-labels (accessed on 13 September 2016).

17. Canadian Food Inspection Agency. Information within the Nutrition Facts Table-Mandatory Information and Serving Size, 2016. Available online: http://www.inspection.gc.ca/food/labelling/food-labellingfor-industry/nutrition-labelling/information-within-the-nutrition-facts-table/eng/1389198568400/ 1389198597278?chap=1 (accessed on 20 May 2016).

18. Australian Government. Australia New Zealand Food Standards Code-Standard 1.2.8-Nutrition Information Requirements, 2016. Available online: https:/ /www.legislation.gov.au/Details/F2016C00162 (accessed on 19 August 2016).

19. European Commission. Regulation (EU) No. 1169/2011 of the European Parliament and of the Council of 25 October 2011 on the Provision of Food Information to Consumers, 2011. Available online: http:/ / eur-lex.europa.eu/legal-content/EN/TXT/HTML/?uri=CELEX:32011R1169\&from=en (accessed on 19 August 2016).

20. Republic of South Africa. Foodstuffs, Cosmetics and Disinfectants Act (54/1972): Regulations Relating to the Labelling and Advertising of Foods: Amendment, 2014. Available online: http://www.gpwonline.co. za/Gazettes/Gazettes/37695_29-5_Health.pdf (accessed on 19 August 2016).

21. Canadian Food Inspection Agency. Food Labelling for Industry: Sweeteners, $2015 . \quad$ Available online: http://www.inspection.gc.ca/food/labelling/food-labelling-for-industry/sweeteners/eng/ 1387749708758/1387750396304?chap=5\#s12c5 (accessed on 13 April 2016). 
22. MacGregor, G.A.; Hashem, K.M. Action on sugar-Lessons from UK salt reduction programme. Lancet 2014, 383, 929-931. [CrossRef]

23. Government of Canada. Consulting Canadians to Modernize and Improve Food Labels-What We Heard, 2014. Available online: http://www.hc-sc.gc.ca/fn-an/alt_formats/pdf/label-etiquet/modernize-reportmoderniser-rapport-eng.pdf (accessed on 25 March 2015).

24. Langlois, K.; Garriguet, D. Sugar consumption among Canadians of all ages. Health Rep. 2011, $22,23-27$. [PubMed]

25. Brisbois, T.; Marsden, S.; Anderson, G.; Sievenpiper, J. Estimated intakes and sources of total and added sugars in the Canadian diet. Nutrients 2014, 6, 1899-1912. [CrossRef] [PubMed]

26. Health Canada. Canadian Community Health Survey Cycle 2.2 Nutrition-A Guide to Accessing and Interpreting the Data, 2006. Available online: http://www.hc-sc.gc.ca/fn-an/surveill/nutrition/commun/ cchs_guide_escc-eng.php (accessed on 10 September 2015).

27. Health Canada. Canadian Nutrient File: Compilation of Canadian Food Composition Data-Users' Guide, 2015. Available online: http://www.hc-sc.gc.ca/fn-an/alt_formats/pdf/nutrition/fiche-nutri-data/user_ guide_utilisation-eng.pdf (accessed on 20 May 2016).

28. Schermel, A.; Emrich, T.; Arcand, J.; Wong, C.; L'Abbé, M. Nutrition marketing on processed food packages in Canada: 2010 food label information program. Appl. Physiol. Nutr. Metab. 2013, 38, 666-672. [CrossRef] [PubMed]

29. Canadian Grocer. Executive Report, Canadian Grocery Industry 2012-2013; Rogers Publishing Limited: Toronto, ON, Canada, 2012.

30. Government of Canada. Food and Drug Regulations (C.R.C., c. 870), 2012. Available online: http:/ /lawslois.justice.gc.ca/PDF/C.R.C.,_c._870.pdf (accessed on 20 May 2016).

31. Sodium Working Group. Sodium Reduction Strategy for Canada: Recommendations of the Sodium Working Group, 2010. Available online: http:/ / www.hc-sc.gc.ca/fn-an/nutrition/sodium/related-info-connexe/ strateg/reduct-strat-eng.php (accessed on 10 March 2015).

32. Health Canada. Nutrient Data: Canadian Nutrient File, 2015. Available online: http://www.healthcanada. gc.ca/cnf (accessed on 10 October 2014).

33. Health Canada. Guidance for the Food Industry on Reducing Sodium in Processed Foods, 2012. Available online: http://www.hc-sc.gc.ca/fn-an/alt_formats/pdf/legislation/guide-ld/2012-sodium-reductionindust-eng.pdf (accessed on 24 August 2014).

34. Hess, J.; Latulippe, M.; Ayoob, K.; Slavin, J. The confusing world of dietary sugars: Definitions, intakes, food sources and international dietary recommendations. Food Funct. 2012, 3, 477-486. [CrossRef] [PubMed]

35. Louie, J.C.Y.; Lei, L.; Rangan, A.M. Reliability of a systematic methodology to estimate added sugars content of foods when applied to a recent Australian food composition database. J. Food Compost. Anal. 2016, 46, 36-42. [CrossRef]

36. Louie, J.C.Y.; Moshtaghian, H.; Boylan, S.; Flood, V.M.; Rangan, A.M.; Barclay, A.W.; Brand-Miller, J.C.; Gill, T.P. A systematic methodology to estimate added sugar content of foods. Eur. J. Clin. Nutr. 2014, 69, 1-8. [CrossRef] [PubMed]

37. United States Department of Agriculture. USDA Database for the Added Sugars Content of Selected Foods, Release 1. Available online: http://www.megaheart.com/pdf/addedsugars.pdf (accessed on 13 September 2016).

38. Pan American Health Organization. Pan American Health Organization Nutrient Profile Model, 2016. Available online: http://iris.paho.org/xmlui/bitstream/handle/123456789/18621/9789275118733_eng.pdf? sequence $=8 \&$ is Allowed $=y$ (accessed on 5 May 2016).

39. Health Canada. Eating Well with Canada's Food Guide, 2011. Available online: http://www.hc-sc.gc.ca/fnan/alt_formats/hpfb-dgpsa/pdf/food-guide-aliment/view_eatwell_vue_bienmang-eng.pdf (accessed on 26 February 2014).

40. Ng, S.W.; Slining, M.M.; Popkin, B.M. Use of caloric and noncaloric sweeteners in US consumer packaged foods, 2005-2009. J. Acad. Nutr. Diet. 2012, 112, 1828-1834. [CrossRef] [PubMed]

41. Goldfein, K.R.; Slavin, J.L. Why sugar is added to food: Food science 101. Compr. Rev. Food Sci. Food Saf. 2015, 14, 644-656. [CrossRef] 
42. United States Department of Agriculture. Scientific Report of the 2015 Dietary Guidelines Advisory Committee, 2015. Available online: http://www.health.gov/dietaryguidelines/2015-scientificreport/PDFs/Scientific-Report-of-the-2015-Dietary-Guidelines-Advisory-Committee.pdf (accessed on 13 February 2016).

43. Gardner, C.; Wylie-Rosett, J.; Gidding, S. Nonnutritive sweeteners: Current use and health perspectives. Circulation 2012, 126, 509-519. [CrossRef] [PubMed]

44. Gortmaker, S.L.; Swinburn, B.A.; Levy, D.; Carter, R.; Mabry, P.L.; Finegood, D.T.; Huang, T.; Marsh, T.; Moodie, M.L. Changing the future of obesity: Science, policy, and action. Lancet 2011, 378, 838-847. [CrossRef]

45. Government of Canada. Regulations Amending the Food and Drug Regulations (Nutrition Labelling, Other Labelling Provisions and Food Colours) [Canada Gazette Part 1 Volume 149 No. 24], 2015. Available online: http:/ /www.gazette.gc.ca/rp-pr/p1/2015/2015-06-13/html/reg1-eng.php (accessed on 17 September 2016).

46. Canadian Food Inspection Agency. Nutrition Labelling Compliance Test, 2014. Available online: http: //www.inspection.gc.ca/english/fssa/labeti/nutricon/nutricone.shtml (accessed on 1 September 2015).

47. Fitzpatrick, L.; Arcand, J.; L'Abbe, M.; Deng, M.; Duhaney, T.; Campbell, N. Accuracy of Canadian food labels for sodium content of food. Nutrients 2014, 6, 3326-3335. [CrossRef] [PubMed]

(C) 2016 by the authors; licensee MDPI, Basel, Switzerland. This article is an open access article distributed under the terms and conditions of the Creative Commons Attribution (CC-BY) license (http://creativecommons.org/licenses/by/4.0/). 